Homology, Homotopy and Applications, vol.11(2), 2009, pp.161-187

\title{
THREE-CROSSED MODULES
}

\author{
Z. ARVASI, T.S. KUZPINARI AND E.Ö. USLU \\ (communicated by Jean-Louis Loday)
}

\begin{abstract}
We introduce the notion of a 3 -crossed module, which extends the notions of a 1-crossed module (Whitehead) and a 2-crossed module (Conduché). We show that the category of 3-crossed modules is equivalent to the category of simplicial groups having a Moore complex of length 3 . We make explicit the relationship with the cat $^{3}$-groups (Loday) and the 3-hypercomplexes (Cegarra-Carrasco), which also model algebraically homotopy 4-types.
\end{abstract}

\section{Introduction}

Crossed modules (or 1-crossed modules) were first defined by Whitehead in [25]. They model connected homotopy 2-types. Conduché [12] in 1984 described the notion of a 2-crossed module as a model of connected 3-types. More generally, Loday [20] gave the foundation of a theory of another algebraic model, which is called cat ${ }^{n}$ groups, for connected $(n+1)$-types. Ellis-Stein $[\mathbf{1 7}]$ showed that cat ${ }^{n}$-groups are equivalent to crossed $n$-cubes. A link between simplicial groups and crossed $n$-cubes were given by Porter $[\mathbf{2 3}]$. Conduché $[\mathbf{1 3}]$ gave a relation between crossed 2-cubes (i.e., crossed squares) and 2-crossed modules. 2-crossed modules were known to be equivalent to that of simplicial groups whose Moore complex has length 2. In $[\mathbf{4}, \mathbf{5}]$, Baues introduced a related notion of a quadratic module. The first author and Ulualan [2] also explored some relations among these algebraic models for (connected) homotopy 3-types.

The most general investigation into the extra structure of the Moore complex of a simplicial group was given by Carrasco-Cegarra in [9] to construct the nonabelian version of the classical Dold-Kan theorem. A much more general context of their work was given by Bourn in [6]. Carrasco and Cegarra arrived at a notion of hypercrossed complexes and proved that the category of such hypercrossed complexes is equivalent to that of simplicial groups. If one truncates hypercrossed complexes at level $n$, throwing away terms of higher dimension, then the resulting $n$-hypercrossed complexes form a category equivalent to the $n$-hyper groupoids of groups given by Duskin [15] and Glenn [18], and give algebraic models for $n$-types. For $n=1$, a 1 hypercrossed complex gives a crossed module, whilst a subcategory of the category of hypercrossed 2-complexes is equivalent to Conduche's category of 2-crossed modules.

Received January 30, 2009, revised July 16, 2009; published on November 12, 2009. 2000 Mathematics Subject Classification: 18D35, 18G30, 18G50, 18G55.

Key words and phrases: crossed module, 2-crossed module, simplicial group, Moore complex.

This article is available at http://www.intlpress.com/HHA/v11/n2/a8/

Copyright (c) 2009, International Press. Permission to copy for private use granted. 
Mutlu-Porter [22] introduced a Peiffer pairing structure within the Moore complexes of a simplicial group. They applied this structure to the study of algebraic models for homotopy types.

In this article we will define the notion of a 3-crossed module as a model for homotopy 4-types. The methods we use are based on ideas of Conduché given in [12] and a Peiffer pairing structure within the Moore complexes of a simplicial group. We prove that the category of 3 -crossed modules is equivalent to that of simplicial groups with Moore complex of length 3 which is equivalent to that of 3-hypercrossed complexes. The main problem with the 3 -hypercrossed complex is difficult to handle intuitively.

The advantages of the notion of a 3-crossed module are the following:

(i) It provides a new algebraic model for (connected) homotopy 4-types;

(ii) It is easy to handle with respect to other models such as the 3-hypercrossed complex;

(iii) It gives a possible way of generalising $n$-crossed modules (or equivalently $n$ groups (see $[\mathbf{2 4}])$ ) which is analogous to a $n$-hypercrossed complex.

(iv) In [5], Baues points out that a "nilpotent" algebraic model for 4-types is not known. 3-crossed modules go some way toward that aim.

\section{Acknowledgements}

This work was partially supported by TÜBITTAK (The Scientific and Technical Research Council of Turkey), and Project Number 107 T542.

\section{Simplicial groups, Moore complexes, Peiffer pairings}

We refer the reader to $[\mathbf{1 4}]$ and $[\mathbf{2 1}]$ for the basic properties of simplicial structures.

\subsection{Simplicial groups}

A simplicial group $\mathbf{G}$ consists of a family of groups $\left\{G_{n}\right\}$ together with face and degeneracy maps $d_{i}^{n}: G_{n} \rightarrow G_{n-1}, 0 \leqslant i \leqslant n,(n \neq 0)$ and $s_{i}^{n}: G_{n-1} \rightarrow G_{n}, 0 \leqslant i \leqslant n$, satisfying the usual simplicial identities given in $[\mathbf{1 4}, \mathbf{2 1}]$. The category of simplicial groups will be denoted by SimpGrp.

Let $\Delta$ denote the category of finite ordinals. For each $k \geqslant 0$ we obtain a subcategory $\Delta_{\leqslant k}$ determined by the objects $[i]$ of $\Delta$ with $i \leqslant k$. A $k$-truncated simplicial group is a functor from $\Delta_{\leqslant k}^{\mathrm{op}}$ to Grp (the category of groups). We will denote the category of $k$-truncated simplicial groups by $\operatorname{Tr}_{k}$ SimpGrp. By a $k$-truncation of a simplicial group, we mean a $k$-truncated simplicial group $\operatorname{tr}_{k} \mathbf{G}$ obtained by forgetting dimensions of order $>k$ in a simplicial group $\mathbf{G}$. Then we have the adjoint situation

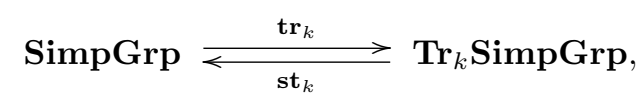

where $\mathbf{s t}_{k}$ is called the $k$-skeleton functor. For detailed definitions see [15]. 


\subsection{The Moore complex}

The Moore complex NG of a simplicial group $\mathbf{G}$ is defined to be the normal chain complex $(\mathbf{N G}, \partial)$ with

$$
N G_{n}=\bigcap_{i=0}^{n-1} \operatorname{Ker} d_{i}
$$

and with the differential $\partial_{n}: N G_{n} \rightarrow N G_{n-1}$ induced from $d_{n}$ by restriction.

The $n$th homotopy group $\pi_{n}(\mathbf{G})$ of $\mathbf{G}$ is the $n$th homology of the Moore complex of $\mathbf{G}$; i.e.,

$$
\pi_{n}(\mathbf{G}) \cong H_{n}(\mathbf{N G}, \partial)=\bigcap_{i=0}^{n} \operatorname{ker} d_{i}^{n} / d_{n+1}^{n+1}\left(\bigcap_{i=0}^{n} \operatorname{ker} d_{i}^{n+1}\right) .
$$

We say that the Moore complex NG of a simplicial group $\mathbf{G}$ is of length $k$ if $\mathbf{N G}_{n}=1$ for all $n \geqslant k+1$. We denote the category of simplicial groups with Moore complex of length $k$ by $\operatorname{SimpGrp} \operatorname{Pr}_{\leqslant k}$.

The Moore complex, NG, carries a hypercrossed complex structure (see Carrasco [9]) from which $\mathbf{G}$ can be rebuilt. We briefly recall some of the aspects of this reconstruction that we will need later.

\subsection{The poset of surjective maps}

The following notation and terminology is derived from [10].

For the ordered set $[n]=\{0<1<\cdots<n\}$, let $\alpha_{i}^{n}:[n+1] \rightarrow[n]$ be the increasing surjective map given by

$$
\alpha_{i}^{n}(j)= \begin{cases}j & \text { if } j \leqslant i, \\ j-1 & \text { if } j>i .\end{cases}
$$

Let $S(n, n-r)$ be the set of all monotone increasing surjective maps from $[n]$ to $[n-r]$. This can be generated from the various $\alpha_{i}^{n}$ by composition. The composition of these generating maps is subject to the following rule:

$$
\alpha_{j} \alpha_{i}=\alpha_{i-1} \alpha_{j}, j<i
$$

This implies that every element $\alpha \in S(n, n-r)$ has a unique expression as $\alpha=$ $\alpha_{i_{1}} \circ \alpha_{i_{2}} \circ \cdots \circ \alpha_{i_{r}}$ with $0 \leqslant i_{1}<i_{2}<\cdots<i_{r} \leqslant n-1$, where the indices $i_{k}$ are the elements of $[n]$ such that $\left\{i_{1}, \ldots, i_{r}\right\}=\{i: \alpha(i)=\alpha(i+1)\}$. We thus can identify $S(n, n-r)$ with the set $\left\{\left(i_{r}, \ldots, i_{1}\right): 0 \leqslant i_{1}<i_{2}<\cdots<i_{r} \leqslant n-1\right\}$. In particular, the single element of $S(n, n)$, defined by the identity map on $[n]$, corresponds to the empty 0-tuple ( ) denoted by $\emptyset_{n}$. Similarly, the only element of $S(n, 0)$ is

$$
(n-1, n-2, \ldots, 0) \text {. }
$$

For all $n \geqslant 0$, let

$$
S(n)=\bigcup_{0 \leqslant r \leqslant n} S(n, n-r) .
$$

We say that $\alpha=\left(i_{r}, \ldots, i_{1}\right)<\beta=\left(j_{s}, \ldots, j_{1}\right)$ in $S(n)$ if $i_{1}=j_{1}, \ldots, i_{k}=j_{k}$ but $i_{k+1}>j_{k+1},(k \geqslant 0)$, or if $i_{1}=j_{1}, \ldots, i_{r}=j_{r}$ and $r<s$. This makes $S(n)$ an ordered 
set. For example,

$$
\begin{aligned}
S(2)= & \left\{\phi_{2}<(1)<(0)<(1,0)\right\} \\
S(3)= & \left\{\phi_{3}<(2)<(1)<(2,1)<(0)<(2,0)<(1,0)<(2,1,0)\right\}, \\
S(4)= & \left\{\phi_{4}<(3)<(2)<(3,2)<(1)<(3,1)<(2,1)<(3,2,1)\right. \\
& <(0)<(3,0)<(2,0)<(3,2,0)<(1,0)<(3,1,0)<(2,1,0)<(3,2,1,0)\} .
\end{aligned}
$$

\subsection{The semidirect decomposition of a simplicial group}

The fundamental idea behind the semidirect decomposition of a simplicial group can be found in Conduché [12]. A detailed investigation of this construction for the case of simplicial groups is given in Carrasco and Cegarra [9].

Given a split extension of groups

$$
1 \longrightarrow K \longrightarrow G \underset{s}{\gtrless} P \longrightarrow 1,
$$

we write $G \cong K \rtimes s(P)$, the semidirect product of the normal subgroup, $K$, with the image of $P$ under the splitting $s$.

Proposition 2.1. If $\boldsymbol{G}$ is a simplicial group, then for any $n \geqslant 0$

$$
\begin{aligned}
G_{n} \cong( & \left.\cdots\left(N G_{n} \rtimes s_{n-1} N G_{n-1}\right) \rtimes \cdots \rtimes s_{n-2} \cdots s_{0} N G_{1}\right) \\
& \rtimes\left(\cdots\left(s_{n-2} N G_{n-1} \rtimes s_{n-1} s_{n-2} N G_{n-2}\right) \rtimes \cdots \rtimes s_{n-1} s_{n-2} \cdots s_{0} N G_{0}\right) .
\end{aligned}
$$

Proof. This is done by the repeated use of the following lemma.

Lemma 2.2. Let $\boldsymbol{G}$ be a simplicial group. Then $G_{n}$ can be decomposed as a semidirect product:

$$
G_{n} \cong \operatorname{Ker} d_{n}^{n} \rtimes s_{n-1}^{n-1}\left(G_{n-1}\right) .
$$

The bracketing and the order of terms in this multiple semidirect product are generated by the sequence

$$
\begin{aligned}
G_{1} \cong & N G_{1} \rtimes s_{0} N G_{0} \\
G_{2} \cong & \left(N G_{2} \rtimes s_{1} N G_{1}\right) \rtimes\left(s_{0} N G_{1} \rtimes s_{1} s_{0} N G_{0}\right) \\
G_{3} \cong & \left(\left(N G_{3} \rtimes s_{2} N G_{2}\right) \rtimes\left(s_{1} N G_{2} \rtimes s_{2} s_{1} N G_{1}\right)\right) \\
& \rtimes\left(\left(s_{0} N G_{2} \rtimes s_{2} s_{0} N G_{1}\right) \rtimes\left(s_{1} s_{0} N G_{1} \rtimes s_{2} s_{1} s_{0} N G_{0}\right)\right)
\end{aligned}
$$

and

$$
\begin{aligned}
& G_{4} \cong(\left(\left(N G_{4} \rtimes s_{3} N G_{3}\right) \rtimes\left(s_{2} N G_{3} \rtimes s_{3} s_{2} N G_{2}\right)\right) \rtimes\left(\left(s_{1} N G_{3} \rtimes s_{3} s_{1} N G_{2}\right)\right. \\
&\left.\left.\rtimes\left(s_{2} s_{1} N G_{2} \rtimes s_{3} s_{2} s_{1} N G_{1}\right)\right)\right) \rtimes s_{0}\left(\text { decomposition of } G_{3}\right) .
\end{aligned}
$$

Note that the term corresponding to $\alpha=\left(i_{r}, \ldots, i_{1}\right) \in S(n)$ is

$$
s_{\alpha}\left(N G_{n-\# \alpha}\right)=s_{i_{r} \cdots i_{1}}\left(N G_{n-\# \alpha}\right)=s_{i_{r}} \cdots s_{i_{1}}\left(N G_{n-\# \alpha}\right),
$$

where $\# \alpha=r$. Hence any element $x \in G_{n}$ can be written in the form

$$
x=y \prod_{\alpha \in S(n)} s_{\alpha}\left(x_{\alpha}\right) \quad \text { with } \quad y \in N G_{n} \quad \text { and } \quad x_{\alpha} \in N G_{n-\# \alpha} .
$$




\subsection{Hypercrossed complex pairings}

In the following we recall from [22] hypercrossed complex pairings. The fundamental idea behind these can be found in Carrasco and Cegarra (cf. [9]). The construction depends on a variety of sources, mainly Conduché $[\mathbf{1 2}]$ and Mutlu and Porter [22]. Define a set $P(n)$ consisting of pairs of elements $(\alpha, \beta)$ from $S(n)$ with $\alpha \cap \beta=\emptyset$ and $\beta<\alpha$, with respect to lexicographic ordering in $S(n)$ where $\alpha=\left(i_{r}, \ldots, i_{1}\right), \beta=\left(j_{s}, \ldots, j_{1}\right) \in S(n)$. The pairings that we will need,

$$
\left\{F_{\alpha, \beta}: N G_{n-\sharp \alpha} \times N G_{n-\sharp \beta} \rightarrow N G_{n}:(\alpha, \beta) \in P(n), n \geqslant 0\right\},
$$

are given as composites by the diagram

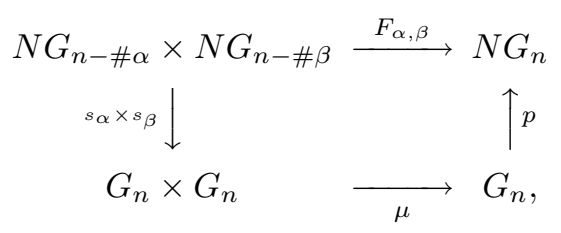

where

$$
\begin{aligned}
& s_{\alpha}=s_{i_{r}}, \ldots, s_{i_{1}}: N G_{n-\sharp \alpha} \rightarrow G_{n}, \\
& s_{\beta}=s_{j_{s}}, \ldots, s_{j_{1}}: N G_{n-\sharp \beta} \rightarrow G_{n} x,
\end{aligned}
$$

and $p: G_{n} \rightarrow N G_{n}$ is defined by the composite projections $p(x)=p_{n-1} \cdots p_{0}(x)$, where $p_{j}(z)=z s_{j} d_{j}(z)^{-1}$ with $j=0,1, \ldots, n-1 . \mu: G_{n} \times G_{n} \rightarrow G_{n}$ is given by a commutator map and $\sharp \alpha$ is the number of the elements in the set of $\alpha$, similarly for $\sharp \beta$. Thus

$$
\begin{aligned}
F_{\alpha, \beta}\left(x_{\alpha}, y_{\beta}\right) & =p \mu\left[\left(s_{\alpha} \times s_{\beta}\right)\left(x_{\alpha}, x_{\beta}\right)\right] \\
& =p\left[\left(s_{\alpha} x_{\alpha} \times s_{\beta} x_{\beta}\right)\right] .
\end{aligned}
$$

Let $N_{n}$ be the normal subgroup of $G_{n}$ generated by elements of the form

$$
F_{\alpha, \beta}\left(x_{\alpha}, y_{\beta}\right),
$$

where $x_{\alpha} \in N G_{n-\sharp \alpha}$ and $y_{\beta} \in N G_{n-\sharp \beta}$.

We illustrate this subgroup for $n=3$ and $n=4$ as follows:

For $n=3$, the possible Peiffer pairings are the following:

$$
F_{(1,0)(2)}, F_{(2,0)(1)}, F_{(0)(2,1)}, F_{(0)(2)}, F_{(1)(2)}, F_{(0)(1)} .
$$

For all $x_{1} \in N G_{1}, y_{2} \in N G_{2}$, the corresponding generators of $N_{3}$ are

$$
\begin{aligned}
& F_{(1,0)(2)}\left(x_{1}, y_{2}\right)=\left[s_{1} s_{0} x_{1}, s_{2} y_{2}\right]\left[s_{2} y_{2}, s_{2} s_{0} x_{1}\right], \\
& F_{(2,0)(1)}\left(x_{1}, y_{2}\right)=\left[s_{2} s_{0} x_{1}, s_{1} y_{2}\right]\left[s_{1} y_{2}, s_{2} s_{1} x_{1}\right]\left[s_{2} s_{1} x_{1}, s_{2} y_{2}\right]\left[s_{2} y_{2}, s_{2} s_{0} x_{1}\right],
\end{aligned}
$$

and for all $x_{2} \in N G_{2}, y_{1} \in N G_{1}$,

$$
F_{(0)(2,1)}\left(x_{2}, y_{1}\right)=\left[s_{0} x_{2}, s_{2} s_{1} y_{1}\right]\left[s_{2} s_{1} y_{1}, s_{1} x_{2}\right]\left[s_{2} x_{2}, s_{2} s_{1} y_{1}\right],
$$


whilst for all $x_{2}, y_{2} \in N G_{2}$,

$$
\begin{aligned}
& F_{(0)(1)}\left(x_{2}, y_{2}\right)=\left[s_{0} x_{2}, s_{1} y_{2}\right]\left[s_{1} y_{2}, s_{1} x_{2}\right]\left[s_{2} x_{2}, s_{2} y_{2}\right], \\
& F_{(0)(2)}\left(x_{2}, y_{2}\right)=\left[s_{0} x_{2}, s_{2} y_{2}\right] \\
& F_{(1)(2)}\left(x_{2}, y_{2}\right)=\left[s_{1} x_{2}, s_{2} y_{2}\right]\left[s_{2} y_{2}, s_{2} x_{2}\right] .
\end{aligned}
$$

For $n=4$, the key pairings are thus the following:

$$
\begin{array}{lllll}
F_{(0)(3,2,1)}, & F_{(3,2,0)(1)}, & F_{(3,1,0)(2)}, & F_{(2,1,0)(3)}, & F_{(3,0)(2,1)}, \\
F_{(2,0)(3,1)}, & F_{(1,0)(3,2)}, & F_{(1)(3,2)}, & F_{(0)(3,2)}, & F_{(0)(3,1)}, \\
F_{(0)(2,1)}, & F_{(3,1)(2)}, & F_{(2,1)(3)}, & F_{(3,0)(2)}, & F_{(3,0)(1)}, \\
F_{(2,0)(3)}, & F_{(2,0)(1)}, & F_{(1,0)(3)}, & F_{(1,0)(2)}, & F_{(2)(3)}, \\
F_{(1)(3)}, & F_{(0)(3)}, & F_{(1)(2)}, & F_{(0)(2)}, & F_{(0)(1)} .
\end{array}
$$

For $x_{1}, y_{1} \in N G_{1}, x_{2}, y_{2} \in N G_{2}$ and $x_{3}, y_{3} \in N G_{3}$ the generator element of the normal subgroup $N_{4}$ can be easily written down from Lemma 2.5 .

Theorem $2.3([\mathbf{2 2}])$. For $n=2,3$ and 4 , let $\mathbf{G}$ be a simplicial group with Moore complex $\mathbf{N G}$ in which $G_{n}=D_{n}$ is the normal subgroup of $G_{n}$ generated by the degenerate elements in dimension $n$. Then

$$
\partial_{n}\left(N G_{n}\right)=\prod_{I, J}\left[K_{I}, K_{J}\right]
$$

for $I, J \subseteq[n-1]$ with

$$
\begin{aligned}
I \cup J & =[n-1], \\
I & =[n-1]-\{\alpha\} \\
J & =[n-1]-\{\beta\},
\end{aligned}
$$

where $(\alpha, \beta) \in P(n)$.

Remark 2.4. In [22], Mutlu and Porter defined the normal subgroup $\partial_{n}\left(N G_{n} \cap D_{n}\right)$ by $F_{\alpha, \beta}$ elements which were first defined by Carrasco in [9]. In [11], Castiglioni and Ladra gave a general proof for the inclusions partially proved by Arvasi and Porter in [1], Arvasi and Akça in [3] and Mutlu and Porter in [22]. Their approach to the problem was different from that of cited works. They have succeeded with a proof, for the case of algebras, over an operad by introducing a different description of the adjoint inverse of the normalization functor $\mathbf{N}: \mathrm{Ab}^{\Delta^{\mathrm{OP}}} \rightarrow \mathrm{Ch}_{\geqslant 0}$. For the case of groups, they then adapted the construction for the adjoint inverse used for algebras to get a simplicial group $G \otimes \Lambda$ from the Moore complex of a simplicial group $G$.

Following the theorem named as Theorem $\mathbf{B}$ in $[\mathbf{2 2}]$, we have

Lemma 2.5. Let $\mathbf{G}$ be a simplicial group with Moore complex $\mathbf{N G}$ of length 3 . Then for the $n=4$ case, the images of $F_{\alpha, \beta}$ elements under $\partial_{4}$, given in the table on the next page, are trivial.

Proof. Since $N G_{4}=1$, by Theorem B in $[\mathbf{2 2}]$ the result is trivial. 


$$
\begin{aligned}
& d_{4}\left(F_{(0)(3,2,1)}\left(x_{3}, x_{1}\right)\right)=\left[s_{0} d_{3} x_{3}, s_{2} s_{1} x_{1}\right]\left[s_{2} s_{1} x_{1}, s_{1} d_{3} x_{3}\right]\left[s_{2} d_{3} x_{3}, s_{2} s_{1} x_{1}\right]\left[s_{2} s_{1} x_{1}, x_{3}\right] \\
& d_{4}\left(F_{(3,2,0)(1)}\left(x_{1}, x_{3}\right)\right)=\left[s_{2} s_{0} x_{1}, s_{1} d_{3} x_{3}\right]\left[s_{1} d_{3} x_{3}, s_{2} s_{1} x_{1}\right]\left[s_{2} s_{1} x_{1}, s_{2} d_{3} x_{3}\right] \\
& {\left[s_{2} d_{3} x_{3}, s_{2} s_{0} x_{1}\right]\left[s_{2} s_{0} x_{1}, x_{3}\right]\left[x_{3}, s_{2} s_{1} x_{1}\right]} \\
& d_{4}\left(F_{(3,1,0)(2)}\left(x_{1}, x_{3}\right)\right)=\left[s_{1} s_{0} x_{1}, s_{2} d_{3} x_{3}\right]\left[s_{2} d_{3} x_{3}, s_{2} s_{0} x_{1}\right]\left[s_{2} s_{0} x_{1}, x_{3}\right]\left[x_{3}, s_{1} s_{0} x_{1}\right] \\
& d_{4}\left(F_{(2,1,0)(3)}\left(x_{1}, x_{3}\right)\right)=\left[s_{2} s_{1} s_{0} d_{1} x_{1}, x_{3}\right]\left[x_{3}, s_{1} s_{0} x_{1}\right] \\
& d_{4}\left(F_{(3,0)(2,1)}\left(x_{2}, y_{2}\right)\right)=\left[s_{0} x_{2}, s_{2} s_{1} d_{2} y_{2}\right]\left[s_{2} s_{1} d_{2} y_{2}, s_{1} x_{2}\right]\left[s_{2} x_{2}, s_{2} s_{1} d_{2} y_{2}\right]\left[s_{1} y_{2}, s_{2} x_{2}\right] \\
& {\left[s_{1} x_{2}, s_{1} y_{2}\right]\left[s_{1} y_{2}, s_{0} x_{2}\right]} \\
& d_{4}\left(F_{(2,0)(3,1)}\left(x_{2}, y_{2}\right)\right)=\left[s_{2} s_{0} d_{2} x_{2}, s_{1} y_{2}\right]\left[s_{1} y_{2}, s_{2} s_{1} d_{2} x_{2}\right]\left[s_{2} s_{1} d_{2} x_{2}, s_{2} y_{2}\right] \\
& {\left[s_{2} y_{2}, s_{2} s_{0} d_{2} x_{2}\right]\left[s_{0} x_{2}, s_{2} y_{2}\right]\left[s_{2} y_{2}, s_{1} x_{2}\right]} \\
& {\left[s_{1} x_{2}, s_{1} y_{2}\right]\left[s_{1} y_{2}, s_{0} x_{2}\right]} \\
& d_{4}\left(F_{(1,0)(3,2)}\left(x_{2}, y_{2}\right)\right)=\left[s_{1} s_{0} d_{2} x_{2}, s_{2} y_{2}\right]\left[s_{2} y_{2}, s_{2} s_{0} d_{2} x_{2}\right]\left[s_{0} x_{2}, s_{2} y_{2}\right] \\
& d_{4}\left(F_{(1)(3,2)}\left(x_{3}, x_{2}\right)\right)=\left[s_{1} d_{3} x_{3}, s_{2} x_{2}\right]\left[s_{2} x_{2}, s_{2} d_{3} x_{3}\right]\left[x_{3}, s_{2} x_{2}\right] \\
& d_{4}\left(F_{(0)(3,2)}\left(x_{3}, x_{2}\right)\right)=\left[s_{0} d_{3} x_{3}, s_{2} x_{2}\right] \\
& d_{4}\left(F_{(3,1)(2)}\left(x_{3}, x_{2}\right)\right)=\left[s_{0} d_{3} x_{3}, s_{1} x_{2}\right]\left[s_{1} x_{2}, s_{1} d_{3} x_{3}\right]\left[s_{2} d_{3} x_{3}, s_{2} x_{2}\right]\left[s_{2} x_{2}, x_{3}\right] \\
& d_{4}\left(F_{(0)(2,1)}\left(x_{3}, x_{2}\right)\right)=\left[s_{0} d_{3} x_{3}, s_{2} s_{1} d_{2} x_{2}\right]\left[s_{2} s_{1} d_{2} x_{2}, s_{1} d_{3} x_{3}\right] \\
& {\left[s_{2} d_{3} x_{3}, s_{2} s_{1} d_{2} x_{2}\right]\left[s_{1} x_{2}, x_{3}\right]} \\
& d_{4}\left(F_{(3,1)(2)}\left(x_{2}, x_{3}\right)\right)=\left[s_{1} x_{2}, s_{2} d_{3} x_{3}\right]\left[s_{2} d_{3} x_{3}, s_{2} x_{2}\right]\left[s_{2} l, x_{3}\right]\left[x_{3}, s_{1} x_{2}\right] \\
& d_{4}\left(F_{(2,1)(3)}\left(x_{2}, x_{3}\right)\right)=\left[s_{2} s_{1} d_{2} x_{2}, x_{3}\right]\left[x_{3}, s_{1} x_{2}\right] \\
& d_{4}\left(F_{(3,0)(2)}\left(x_{2}, x_{3}\right)\right)=\left[s_{0} x_{2}, s_{2} d_{3} x_{3}\right]\left[x_{3}, s_{0} x_{2}\right] \\
& d_{4}\left(F_{(3,0)(1)}\left(x_{2}, x_{3}\right)\right)=\left[s_{0} x_{2}, s_{1} d_{3} x_{3}\right]\left[s_{1} d_{3} x_{3}, s_{1} x_{2}\right]\left[s_{2} x_{2}, s_{2} d_{3} x_{3}\right]\left[x_{3}, s_{2} x_{2}\right] \\
& d_{4}\left(F_{(2,0)(3)}\left(x_{2}, x_{3}\right)\right)=\left[s_{2} s_{0} d_{2} x_{2}, x_{3}\right]\left[x_{3}, s_{0} x_{2}\right] \\
& d_{4}\left(F_{(2,0)(1)}\left(x_{2}, x_{3}\right)\right)=\left[s_{2} s_{0} d_{2} x_{2}, s_{1} d_{3} x_{3}\right]\left[s_{1} d_{3} x_{3}, s_{2} s_{1} d_{2} x_{2}\right]\left[s_{2} s_{1} d_{2} x_{2}, s_{2} d_{3} x_{3}\right] \\
& {\left[s_{2} d_{3} x_{3}, s_{2} s_{0} d_{2} x_{2}\right]\left[s_{0} x_{2}, x_{3}\right]\left[x_{3}, s_{1} x_{2}\right]} \\
& d_{4}\left(F_{(1,0)(3)}\left(x_{2}, x_{3}\right)\right)=\left[s_{1} s_{0} d_{2} x_{2}, x_{3}\right] \\
& d_{4}\left(F_{(1,0)(2)}\left(x_{2}, x_{3}\right)\right)=\left[s_{1} s_{0} d_{2} x_{2}, s_{2} d_{3} x_{3}\right]\left[s_{2} d_{3} x_{3}, s_{2} s_{0} d_{2} x_{2}\right]\left[s_{0} x_{2}, x_{3}\right] \\
& d_{4}\left(F_{(2)(3)}\left(x_{3}, y_{3}\right)\right)=\left[s_{2} d_{3} x_{3}, y_{3}\right]\left[y_{3}, x_{3}\right] \\
& d_{4}\left(F_{(1)(3)}\left(x_{3}, y_{3}\right)\right)=\left[s_{1} d_{3} x_{3}, y_{3}\right] \\
& d_{4}\left(F_{(0)(3)}\left(x_{3}, y_{3}\right)\right) \quad=\left[s_{0} d_{3} x_{3}, y_{3}\right] \\
& d_{4}\left(F_{(1)(2)}\left(x_{3}, y_{3}\right)\right)=\left[s_{1} d_{3} x_{3}, s_{2} d_{3} y_{3}\right]\left[s_{2} d_{3} y_{3}, s_{2} d_{3} x_{3}\right]\left[x_{3}, y_{3}\right] \\
& d_{4}\left(F_{(0)(2)}\left(x_{3}, y_{3}\right)\right)=\left[s_{0} d_{3} x_{3}, s_{2} d_{3} y_{3}\right] \\
& d_{4}\left(F_{(0)(1)}\left(x_{3}, y_{3}\right)\right)=\left[s_{0} d_{3} x_{3}, s_{1} d_{3} y_{3}\right]\left[s_{1} d_{3} y_{3}, s_{1} d_{3} x_{3}\right]\left[s_{2} d_{3} x_{3}, s_{2} d_{3} y_{3}\right]\left[y_{3}, x_{3}\right]
\end{aligned}
$$

where $x_{3}, y_{3} \in N G_{3}, x_{2}, y_{2} \in N G_{2}, x_{1} \in N G_{1}$. 


\section{2-crossed modules}

The notion of a crossed module is an efficient algebraic tool to handle connected spaces with only the first homotopy groups nontrivial, up to homotopy.

A crossed module is a group homomorphism $\partial: M \rightarrow P$ together with an action of $P$ on $M$, written ${ }^{p} m$ for $p \in P$ and $m \in M$, satisfying the conditions:

CM1) $\partial$ is $P$-equivariant; i.e., for all $p \in P, m \in M$,

$$
\partial\left({ }^{p} m\right)=p \partial(m) p^{-1} \text {. }
$$

CM2) (Peiffer identity). For all $m, m^{\prime} \in M$,

$$
{ }^{\partial m} m^{\prime}=m m^{\prime} m^{-1} \text {. }
$$

We will denote such a crossed module by $(M, P, \partial)$.

A morphism of a crossed module from $(M, P, \partial)$ to $\left(M^{\prime}, P^{\prime}, \partial^{\prime}\right)$ is a pair of group homomorphisms

$$
\phi: M \longrightarrow M^{\prime}, \psi: P \longrightarrow P^{\prime}
$$

such that $\phi\left({ }^{p} m\right)={ }^{\psi(p)} \phi(m)$ and $\partial^{\prime} \phi(m)=\psi \partial(m)$.

We thus get a category XMod of crossed modules.

Examples of crossed modules:

1) Any normal subgroup $N \unlhd P$ gives rise to a crossed module namely the inclusion map, $i: N \hookrightarrow P$. Conversely, given any crossed module $\partial: M \longrightarrow P, \operatorname{Im} \partial$ is a normal subgroup of $P$.

2) Given any $P$-module $M$, the trivial map1: $M \longrightarrow P$, which maps everything to 1 in $P$, is a crossed module. Conversely, if $\partial: M \rightarrow P$ is a crossed module, then ker $\partial$ is central in $M$ and inherits a natural $P$-module structure from the $P$-action on $M$.

The following definition of a 2-crossed module is equivalent to that given by Conduché $([\mathbf{1 2}])$.

A 2-crossed module of groups consists of a complex of groups

$$
L \stackrel{\partial_{2}}{\longrightarrow} M \stackrel{\partial_{1}}{\longrightarrow} N
$$

together with

(a) actions of $N$ on $M$ and $L$ so that $\partial_{2}, \partial_{1}$ are morphisms of $N$-groups, and

(b) an $N$-equivariant function

$$
\{, \quad\}: M \times M \longrightarrow L
$$

called a Peiffer lifting. 
This data must satisfy the following axioms:

\begin{tabular}{|c|c|}
\hline $\begin{array}{l}\text { 2CM1) } \\
\text { 2CM2) }\end{array}$ & $\begin{aligned} \partial_{2}\left\{m, m^{\prime}\right\} & =\left({ }^{\partial_{1} m} m^{\prime}\right) m m^{\prime-1} m^{-1} \\
\left\{\partial_{2} l, \partial_{2} l^{\prime}\right\} & =\left[l^{\prime}, l\right] .\end{aligned}$ \\
\hline 2CM3) & $\left\{m m^{\prime}, m^{\prime \prime}\right\}={ }^{\partial_{1} m}\left\{m^{\prime}, m^{\prime \prime}\right\}\left\{m, m^{\prime} m^{\prime \prime} m^{-1}\right\}$ \\
\hline & (ii) $\quad\left\{m, m^{\prime} m^{\prime \prime}\right\}=\left\{m, m^{\prime}\right\}^{m m^{\prime} m^{-1}}\left\{m, m^{\prime \prime}\right\}$ \\
\hline 2CM4) & $\left\{m, \partial_{2} l\right\}\left\{\partial_{2} l, m\right\}={ }^{\partial_{1} m} l l^{-1}$ \\
\hline 2CM5) & ${ }^{n}\left\{m, m^{\prime}\right\}=\left\{{ }^{n} m,{ }^{n} m^{\prime}\right\}$ \\
\hline
\end{tabular}

for all $l, l^{\prime} \in L, m, m^{\prime}, m^{\prime \prime} \in M$ and $n \in N$.

Here we have used ${ }^{m} l$ as a shorthand for $\left\{\partial_{2} l, m\right\} l$ in condition 2CM3)(ii) where $l$ is $\left\{m, m^{\prime \prime}\right\}$ and $m$ is $m m^{\prime}(m)^{-1}$. This gives a new action of $M$ on $L$. Using this notation, we can split 2CM4) into two pieces, the first of which is tautologous:

2CM4)

(a) $\left\{\partial_{2} l, m\right\}={ }^{m} l(l)^{-1}$,

(b) $\left\{m, \partial_{2} l\right\}=\left({ }^{\partial_{1} m} l\right)\left({ }^{m} l^{-1}\right)$.

The old action of $M$ on $L$, via $\partial_{1}$ and the $N$-action on $L$, is, in general, distinct from this second action with $\left\{m, \partial_{2} l\right\}$ measuring the difference (by 2CM4)(b)). An easy argument using 2CM2) and 2CM4)(b) shows that with this action, ${ }^{m} l$, of $M$ on $L,\left(L, M, \partial_{2}\right)$ becomes a crossed module. A morphism of 2-crossed modules can be defined in an obvious way. We thus define the category of 2-crossed modules denoting it by $\mathbf{X}_{2}$ Mod.

A crossed square as defined by D. Guin-Waléry and J.-L. Loday in [19] (see also [8, 20]) can be seen as a mapping cone in [13]. Furthermore, 2-crossed modules are related to simplicial groups. This relation can be found in $[\mathbf{1 2}, \mathbf{2 2}]$.

Theorem 3.1. The category $\mathbf{X}_{2}$ Mod of 2-crossed modules is equivalent to the category of SimpGrp $\mathbf{S}_{\leqslant 2}$ simplicial groups with Moore complex of length 2.

\section{3-crossed modules}

In the following we will define the category of 3-crossed modules. First of all we adapt ideas from Conduché's method given in [12]. He gave some equalities by using the semi-direct decomposition of a simplicial group, but these are exactly the images of Peiffer pairings $F_{\alpha, \beta}$ under $\partial_{3}$ for $n=3$ case defined in [22]. The difference of our method is to use $F_{\alpha, \beta}$ instead of semi-direct decomposition. Thus we will define similar equalities for $n=4$ and get the axioms of a 3 -crossed module.

Let $\mathbf{G}$ be a simplicial group with Moore complex of length 3 and $N G_{0}=N, N G_{1}$ $=M, N G_{2}=L, N G_{3}=K$. Thus we have a group complex

$$
K \stackrel{\partial_{3}}{\longrightarrow} L \stackrel{\partial_{2}}{\longrightarrow} M \stackrel{\partial_{1}}{\longrightarrow} N \text {. }
$$


Let the actions of $N$ on $K, L, M, M$ on $L, K$ and $L$ on $K$ be as follows:

$$
\begin{aligned}
{ }^{n} m & =s_{0} n(m) s_{0} n^{-1}, \\
{ }^{n} l & =s_{1} s_{0} n(l) s_{1} s_{0} n^{-1}, \\
{ }^{n} k & =s_{2} s_{1} s_{0} n(k) s_{2} s_{1} s_{0} n^{-1} \\
{ }^{m} l & =s_{1} m(l) s_{1} m^{-1}, \\
{ }^{m} k & =s_{2} s_{1} m(k) s_{2} s_{1} m^{-1}, \\
l \cdot k & =s_{2} l(k) s_{2} l^{-1} .
\end{aligned}
$$

Using the table on page 167 , since

$$
\begin{aligned}
{\left[s_{1} s_{0} m s_{2} s_{1} \partial_{1} m, k\right] } & =1, \\
{\left[s_{1} l s_{2} s_{1} \partial_{2} l, k\right] } & =1, \\
{\left[k^{\prime}, k^{-1} s_{2} \partial_{3} k\right] } & =1,
\end{aligned}
$$

we get

$$
\begin{aligned}
\partial_{1} m k & =s_{1} s_{0} m(k) s_{1} s_{0} m^{-1}, \\
\partial_{2} l & =s_{1} l(k) s_{1} l^{-1}, \\
\partial_{3} k \cdot k^{\prime} & =k\left(k^{\prime}\right) k^{-1},
\end{aligned}
$$

and using the simplicial identities we get

$$
\partial_{3}(l \cdot k)=\partial_{3}\left(s_{2} l(k) s_{2} l^{-1}\right)=\partial_{3} s_{2} l\left(\partial_{3} k\right) s_{2} l^{-1}=l\left(\partial_{3} k\right) l^{-1} .
$$

Thus $\partial_{3}: K \rightarrow L$ is a crossed module.

The Peiffer liftings given in the definition below are the $F_{\alpha, \beta}$ pairings for the case $n=3$ defined in $[\mathbf{9}]$.

Definition 4.1. Let $K \stackrel{\partial_{3}}{\longrightarrow} L \stackrel{\partial_{2}}{\longrightarrow} M \stackrel{\partial_{1}}{\longrightarrow} N$ be a group complex defined above. We define the Peiffer liftings as follows:

$$
\begin{aligned}
& \{,\}: \quad M \times M \quad \longrightarrow L \\
& \left\{m, m^{\prime}\right\}=\left[s_{1} m, s_{1} m^{\prime}\right]\left[s_{1} m^{\prime}, s_{0} m\right] \\
& \{,\}_{(1)(0)}: \quad L \times L \quad \longrightarrow K \\
& \left\{l, l^{\prime}\right\}_{(1)(0)}=\left[s_{2} l^{\prime}, s_{2} l\right]\left[s_{1} l, s_{1} l^{\prime}\right]\left[s_{1} l^{\prime}, s_{0} l\right] \\
& \{,\}_{(2)(1)}: \quad L \times L \quad \longrightarrow K \\
& \left\{l, l^{\prime}\right\}_{(2)(1)}=\left[s_{2} l, s_{2} l^{\prime}\right]\left[s_{2} l^{\prime}, s_{1} l\right] \\
& \{,\}_{(0)(2)}: \quad L \times L \quad \longrightarrow K \\
& \left\{l, l^{\prime}\right\}_{(0)(2)}=\left[s_{2} l^{\prime}, s_{0} l\right]
\end{aligned}
$$

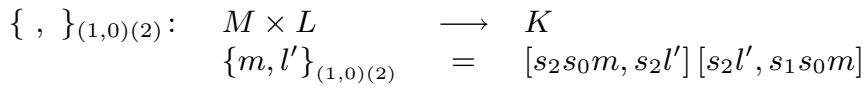

$$
\begin{aligned}
& \{,\}_{(2,0)(1)}: M \times L \quad \longrightarrow K
\end{aligned}
$$

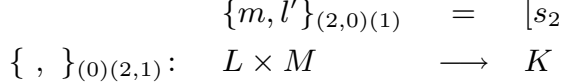

$$
\begin{aligned}
& \left\{l^{\prime}, m\right\}_{(0)(2,1)}=\left[s_{2} s_{1} m, s_{2} l^{\prime}\right]\left[s_{1} l^{\prime}, s_{2} s_{1} m\right]\left[s_{2} s_{1} m, s_{0} l^{\prime}\right]
\end{aligned}
$$

where $m, m^{\prime} \in M, l, l^{\prime} \in L$. 
Then using the table on page 167 we get the following identities:

$$
\begin{array}{ll}
\left\{m, \partial_{3} k\right\}_{(1,0)(2)} & =\left\{m, \partial_{3} k\right\}_{(2,0)(1)}{ }^{m}(k)^{\partial_{1} m}\left(k^{-1}\right) \\
\left\{\partial_{3} k, m\right\}_{(0)(2,1)} & =m(k) k^{-1} \\
\left\{m, \partial_{3} k\right\}_{(1,0)(2)} & =\left\{l, l^{\prime}\right\}_{(2)(1)}^{-1}\left\{l^{\prime}, l\right\}_{(1)(0)} \\
\left\{l^{\prime}, \partial_{2} l\right\}_{(0)(2,1)} & =\left\{l, l^{\prime}\right\}_{(0)(2)}^{-1}\left[l^{\prime}, l\right]_{\left(\left\{l, l^{\prime}\right\}_{(2)(1)}\right)\left\{l, l^{\prime}\right\}_{(1)(0)}} \\
\left\{\partial_{2} l, l^{\prime}\right\}_{(2,0)(1)} \partial_{1} m\left(k^{-1}\right) \\
\left\{\partial_{2} l, l^{\prime}\right\}_{(1,0)(2)} & =\left(\left\{l, l^{\prime}\right\}_{(0)(2)}\right)^{-1} \\
\left\{l, l^{\prime} l^{\prime \prime}\right\}_{(2)(1)} & =\left\{l, l^{\prime}\right\}_{(2)(1)} \partial l l^{\prime} .\left\{l, l^{\prime \prime}\right\}_{(2)(1)} \\
\left\{l l^{\prime}, l^{\prime \prime}\right\}_{(2)(1)} & =l \cdot\left\{l^{\prime}, l^{\prime \prime}\right\}_{(2)(1)}\left\{l, l^{\prime} l^{\prime \prime}\right\}_{(2)(1)} \\
\partial_{3}\left(\left\{l, l^{\prime}\right\}_{(1)(0)}\right) & =\left[l, l^{\prime}\left\{\partial_{2} l, \partial_{2} l^{\prime}\right\}\right. \\
\partial_{3}\left(\left\{l, l^{\prime}\right\}_{(2)(1)}\right) & =l l^{\prime} l^{-1}\left(\partial_{2} l l^{\prime}\right)^{-1} \\
\partial_{3}\left(\left\{l, l^{\prime}\right\}_{(0)(2)}\right) & =\partial_{3}\left(\left\{\partial_{2} l, l^{\prime}\right\}_{(1,0)(2)}\right)^{-1} \\
\partial_{3}\{l, m\}_{(0)(2,1)} & =l^{-1}\left\{\partial_{2} l, m\right\} \\
\partial_{3}\{m, l\}_{(2,0)(1)} & =\partial_{3}\{m, l\}_{(1,0)(2)} \partial_{1} m l^{m}\left(l^{-1}\right)\left\{m, \partial_{2} l\right\} \\
\left\{\partial_{3} k, l\right\}_{(2)(1)}\left\{l, \partial_{3} k\right\}_{(2)(1)} & =k\left(\partial_{2} l\left(k^{-1}\right)\right) \\
\left\{\partial_{3} k, l\right\}_{(1)(0)}\left\{l, \partial_{3} k\right\}_{(1)(0)} & =1 \\
\left\{\partial_{3} k, \partial_{3} k^{\prime}\right\}_{(2)(1)} & =\left[k, k^{\prime}\right] \\
\left\{\partial_{3} k, \partial_{3} k^{\prime}\right\}_{(1)(0)} & =\left[k^{\prime}, k\right] \\
\left\{\partial_{3} k, l^{\prime}\right\}_{(0)(2)} & =1 \\
\left\{\partial_{2} l, \partial_{3} k\right\}_{(1,0)(2)} & =\left\{l, \partial_{3} k\right\}_{(0)(2)}^{-1} \\
\left\{\partial_{2} l, \partial_{3} k\right\}_{(2,0)(1)} & =\left\{l, \partial_{3} k\right\}_{(0)(2)} k\left(\partial_{2} l\left(k^{-1}\right)\right) \\
\left\{\partial_{3} k, \partial_{2} l\right\}_{(0)(2,1)} & =\partial_{2} l k k^{-1}
\end{array}
$$

Table 1

$$
\begin{array}{ll}
{ }^{n}\left\{m, m^{\prime}\right\} & =\left\{{ }^{n} m,{ }^{n} m^{\prime}\right\} \\
{ }^{n}\left\{l, l^{\prime}\right\}_{(1)(0)} & =\left\{{ }^{n} l,{ }^{n} l^{\prime}\right\}_{(1)(0)} \\
{ }^{n}\left\{l, l^{\prime}\right\}_{(2)(1)} & =\left\{{ }^{n} l,{ }^{n} l^{\prime}\right\}_{(2)(1)} \\
{ }^{n}\left\{l, l^{\prime}\right\}_{(0)(2)} & =\left\{{ }^{n} l,{ }^{n} l^{\prime}\right\}_{(0)(2)} \\
{ }^{n}\left\{m, l^{\prime}\right\}_{(1,0)(2)} & =\left\{{ }^{n} m,{ }^{n} l^{\prime}\right\}_{(1,0)(2)} \\
{ }^{n}\left\{m, l^{\prime}\right\}_{(2,0)(1)} & =\left\{{ }^{n} m,{ }^{n} l^{\prime}\right\}_{(2,0)(1)} \\
{ }^{n}\left\{l^{\prime}, m\right\}_{(0)(2,1)} & =\left\{{ }^{n} l^{\prime},{ }^{n} m\right\}_{(0)(2,1)}
\end{array}
$$

Table 2 


$$
\begin{aligned}
& { }^{m}\left\{m^{\prime}, m^{\prime \prime}\right\} \quad=\quad m\left\{m^{\prime},{ }^{m} m^{\prime \prime}\right\} \\
& \left.{ }^{m}\left\{l, l^{\prime}\right\}_{(1)(0)}={ }^{m} l,{ }^{m} l^{\prime}\right\}_{(1)(0)} \\
& { }^{m}\left\{l, l^{\prime}\right\}_{(2)(1)}=\left\{{ }^{m} l,{ }^{m} l^{\prime}\right\}_{(2)(1)} \\
& { }^{m}\left\{l, l^{\prime}\right\}_{(0)(2)}=\left\{{ }^{m} l,{ }^{m} l^{\prime}\right\}_{(0)(2)} \\
& { }^{m}\left\{m, l^{\prime}\right\}_{(1,0)(2)}=\left\{{ }^{m} m,{ }^{m} l^{\prime}\right\}_{(1,0)(2)} \\
& \left.{ }^{m}\left\{m, l^{\prime}\right\}_{(2,0)(1)}={ }^{m} m,{ }^{m} l^{\prime}\right\}_{(2,0)(1)} \\
& \left.{ }^{m}\left\{l^{\prime}, m\right\}_{(0)(2,1)}={ }^{m} l^{\prime},{ }^{m} m\right\}_{(0)(2,1)}
\end{aligned}
$$

Table 3

where $m, m^{\prime}, m^{\prime \prime} \in M, l, l^{\prime} \in L, k, k^{\prime} \in K$. From these results, all liftings are $N$ - and $M$-equivariant.

Definition 4.2. A 3-crossed module consists of a complex of groups

$$
K \stackrel{\partial_{3}}{\longrightarrow} L \stackrel{\partial_{2}}{\longrightarrow} M \stackrel{\partial_{1}}{\longrightarrow} N
$$

together with an action of $N$ on $K, L$ and $M$, an action of $M$ on $K$ and $L$, an action of $L$ on $K$ so that $\partial_{3}, \partial_{2}, \partial_{1}$ are morphisms of $N, M$-groups, and $M, N$-equivariant liftings

$$
\begin{aligned}
& \{,\}_{(1)(0)}: L \times L \longrightarrow K, \quad\{,\}_{(0)(2)}: L \times L \longrightarrow K, \quad\{,\}_{(2)(1)}: L \times L \longrightarrow K, \\
& \{,\}_{(1,0)(2)}: M \times L \longrightarrow K, \quad\{,\}_{(2,0)(1)}: M \times L \longrightarrow K, \\
& \{,\}_{(0)(2,1)}: L \times M \longrightarrow K, \quad\{,\}: M \times M \longrightarrow L
\end{aligned}
$$

called 3-dimensional Peiffer liftings. This data must satisfy the following axioms:

$$
\begin{aligned}
& \text { 3CM1) } \quad K \stackrel{\partial_{3}}{\longrightarrow} L \stackrel{\partial_{2}}{\longrightarrow} M \text { is a 2-crossed module with the Peiffer lifting }\{,\}(2,1) \\
& \text { 3CM2) } \quad\left\{m, \partial_{3} k\right\}_{(1,0)(2)}=\left\{m, \partial_{3} k\right\}_{(2,0)(1)}^{m}(k)^{\partial_{1} m}\left(k^{-1}\right) \\
& \text { 3CM3) } \quad\left\{\partial_{3} k, m\right\}_{(0)(2,1)}={ }^{m}(k) k^{-1} \\
& \text { 3CM4) } \quad\left\{m, \partial_{3} k\right\}_{(1,0)(2)}=\left\{m, \partial_{3} k\right\}_{(2,0)(1)}\left\{\partial_{3} k, m\right\}_{(0)(2,1)} k^{\partial_{1} m}\left(k^{-1}\right) \\
& \text { 3CM5) } \quad\left\{l^{\prime}, \partial_{2} l\right\}_{(0)(2,1)}=\left\{l, l^{\prime}\right\}_{(2)(1)}^{-1}\left\{l^{\prime}, l\right\}_{(1)(0)} \\
& \text { 3CM6) } \quad\left\{\partial_{2} l, l^{\prime}\right\}_{(2,0)(1)}=\left\{l, l^{\prime}\right\}_{(0)(2)}^{-1}\left[l^{\prime}, l\right]\left(\left\{l, l^{\prime}\right\}_{(2)(1)}\right)\left\{l, l^{\prime}\right\}_{(1)(0)} \\
& \text { 3CM7) } \quad\left\{\partial_{2} l, l^{\prime}\right\}_{(1,0)(2)}=\left(\left\{l, l^{\prime}\right\}_{(0)(2)}\right)^{-1} \\
& \text { 3CM8) } \quad \partial_{3}\left(\left\{l, l^{\prime}\right\}_{(1)(0)}\right)=\left[l, l^{\prime}\right]\left\{\partial_{2} l, \partial_{2} l^{\prime}\right\} \\
& \text { 3CM9) } \quad \partial_{3}\left(\left\{l, l^{\prime}\right\}_{(0)(2)}\right)=\partial_{3}\left(\left\{\partial_{2} l, l^{\prime}\right\}_{(1,0)(2)}\right)^{-1} \\
& \text { 3CM10) } \quad \partial_{3}\{l, m\}_{(0)(2,1)}={ }^{m} l l^{-1}\left\{\partial_{2} l, m\right\} \\
& \text { 3CM11) } \quad \partial_{3}\{m, l\}_{(2,0)(1)}=\partial_{3}\{m, l\}_{(1,0)(2)}{ }^{\partial_{1} m} l^{m}\left(l^{-1}\right)\left\{m, \partial_{2} l\right\} \\
& \text { 3CM12a) } \quad\left\{\partial_{3} k, l\right\}_{(1)(0)}=\left({ }^{l} k\right) k^{-1} \\
& \text { 3CM12b) } \quad\left\{l, \partial_{3} k\right\}_{(1)(0)}=k\left({ }^{l} k\right)^{-1} \\
& \text { 3CM13) } \quad\left\{\partial_{3} k, \partial_{3} k^{\prime}\right\}_{(1)(0)}=\left[k^{\prime}, k\right]
\end{aligned}
$$




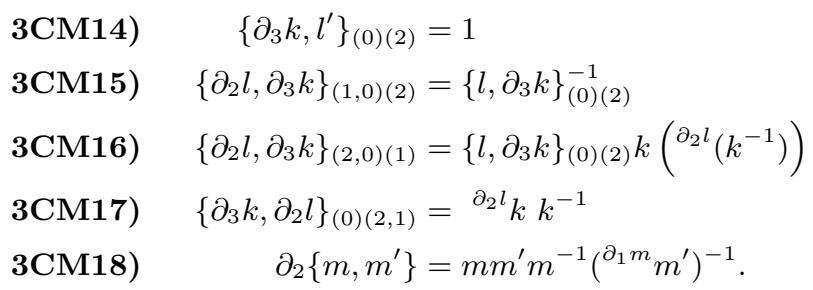

We denote such a 3 -crossed module by $\left(K, L, M, N, \partial_{3}, \partial_{2}, \partial_{1}\right)$.

A morphism of 3 -crossed modules of groups may be pictured by the diagram

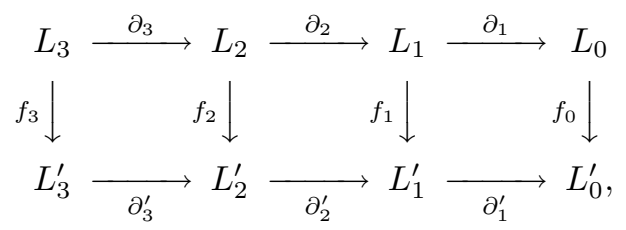

where

$$
f_{1}\left({ }^{n} m\right)={ }^{\left(f_{0}(n)\right)} f_{1}(m), f_{2}\left({ }^{n} l\right)={ }^{\left(f_{0}(n)\right)} f_{2}(l), f_{3}\left({ }^{n} k\right)={ }^{\left(f_{0}(n)\right)} f_{3}(k) .
$$

We require the following equations to hold: for $\{,\}_{(0)(2)},\{,\}_{(2)(1)}$ and $\{,\}_{(1)(0)}$,

$$
\{,\} f_{2} \times f_{2}=f_{3}\{,\}
$$

for $\{,\}_{(1,0)(2)}$ and $\{,\}_{(2,0)(1)}$,

$$
\{,\} f_{1} \times f_{2}=f_{3}\{,\}
$$

for $\{,\}_{(0)(2,1)}$,

$$
\{,\} f_{2} \times f_{1}=f_{3}\{,\}
$$

and for $\{$,$\} ,$

$$
\{,\} f_{1} \times f_{1}=f_{2}\{,\}
$$

for all $k \in K, l \in L, m \in M$ and $n \in N$. These compose in an obvious way. We thus can define the category of 3 -crossed modules, denoting it by $\mathbf{X}_{3}$ Mod.

\section{Applications}

\subsection{Simplicial groups}

As an application we consider in detail the relation between simplicial groups and 3 -crossed modules.

Proposition 5.1. Let $\mathbf{G}$ be a simplicial group with Moore complex NG. Then the group complex

$$
N G_{3} / \partial_{4}\left(N G_{4} \cap D_{4}\right) \stackrel{\bar{\partial}_{3}}{\longrightarrow} N G_{2} \stackrel{\partial_{2}}{\longrightarrow} N G_{1} \stackrel{\partial_{1}}{\longrightarrow} N G_{0}
$$


is a 3-crossed module with the Peiffer liftings defined below:

$$
\begin{aligned}
& \{,\}: N G_{1} \times N G_{1} \longrightarrow N G_{2} \\
& \left\{x_{1}, y_{1}\right\} \longmapsto\left[s_{0} x_{1}, s_{1} y_{1}\right]\left[s_{1} y_{1}, s_{1} x_{1}\right], \\
& \{,\}_{(1)(0)}: N G_{2} \times N G_{2} \longrightarrow N G_{3} / \partial_{4}\left(N G_{4} \cap D_{4}\right) \\
& \left\{x_{2}, y_{2}\right\} \longmapsto \overline{\left(\left[s_{0} x_{2}, s_{1} y_{2}\right]\left[s_{1} y_{2}, s_{1} x_{2}\right]\left[s_{2} x_{2}, s_{2} y_{2}\right]\right)}, \\
& \{,\}_{(2)(1)}: N G_{2} \times N G_{2} \longrightarrow N G_{3} / \partial_{4}\left(N G_{4} \cap D_{4}\right) \\
& \left\{x_{2}, y_{2}\right\} \longmapsto \overline{\left(\left[s_{1} x_{2}, s_{2} y_{2}\right]\left[s_{2} y_{2}, s_{2} x_{2}\right]\right)}, \\
& \{,\}_{(0)(2)}: N G_{2} \times N G_{2} \longrightarrow N G_{3} / \partial_{4}\left(N G_{4} \cap D_{4}\right) \\
& \left\{x_{2}, y_{2}\right\} \longmapsto \overline{\left(\left[s_{0} x_{2}, s_{2} y_{2}\right]\right)}, \\
& \{,\}_{(1,0)(2)}: N G_{1} \times N G_{2} \longrightarrow N G_{3} / \partial_{4}\left(N G_{4} \cap D_{4}\right) \\
& \left\{x_{1}, y_{2}\right\} \longmapsto \overline{\left(\left[s_{1} s_{0} x_{1}, s_{2} y_{2}\right]\left[s_{2} y_{2}, s_{2} s_{0} x_{1}\right]\right)}, \\
& \left.\{,\}_{(2,0)(1)}: N G_{1} \times N G_{2} \longrightarrow N G_{3} / \partial_{4}\left(N G_{4} \cap D\right) 4\right) \\
& \left\{x_{1}, y_{2}\right\} \longmapsto \overline{\left(\left[s_{2} s_{0} x_{1}, s_{1} y_{2}\right]\left[s_{1} y_{2}, s_{2} s_{1} x_{1}\right]\left[s_{2} s_{1} x_{1}, s_{2} y_{2}\right]\left[s_{2} y_{2}, s_{2} s_{0} x_{1}\right]\right)} \\
& \{,\}_{(0)(2,1)}: N G_{2} \times N G_{1} \longrightarrow N G_{3} / \partial_{4}\left(N G_{4} \cap D_{4}\right) \\
& \left\{y_{2}, x_{1}\right\} \longmapsto \overline{\left(\left[s_{0} y_{2}, s_{2} s_{1} x_{1}\right]\left[s_{2} s_{1} x_{1}, s_{1} y_{2}\right]\right)\left[s_{2} y_{2}, s_{2} s_{1} x_{1}\right] \mid}
\end{aligned}
$$

(The elements denoted by $\overline{[,]}$ are cosets in $N G_{3} / \partial_{4}\left(N G_{4} \cap D_{4}\right)$ and are given by the elements in $N G_{3}$.)

Proof. The proof is given in Appendix A..

Theorem 5.2. The category of 3-crossed modules is equivalent to the category of simplicial groups with Moore complex of length 3.

Proof. Let $\mathbf{G}$ be a simplicial group with Moore complex of length 3 . In the above proposition we showed that the group complex

$$
N G_{3} \stackrel{\partial_{3}}{\longrightarrow} N G_{2} \stackrel{\partial_{2}}{\longrightarrow} N G_{1} \stackrel{\partial_{1}}{\longrightarrow} N G_{0}
$$

is a 3 -crossed module. Since the Moore complex is of length $3, N G_{4} \cap D_{4}=1$, so $\partial_{4}\left(N G_{4} \cap D_{4}\right)=1$. Thus we can take $N G_{3}$ instead of $\left.N G_{3} / \partial_{4}\left(N G_{4} \cap D_{4}\right)\right)$. Finally, there is a functor

$$
\Im_{3}: \operatorname{SimpGrp}_{\leqslant 3} \longrightarrow \mathbf{X}_{3} \operatorname{Mod}
$$

from the category of simplicial groups with Moore complex of length 3 to the category of 3-crossed modules. Conversely, let

$$
K \stackrel{\partial_{3}}{\longrightarrow} L \stackrel{\partial_{2}}{\longrightarrow} M \stackrel{\partial_{1}}{\longrightarrow} N
$$

be a 3 -crossed module. Let $H_{0}=N$. By the action of $N$ on $M$ obtain the $H_{1}=M \rtimes N$ 
semidirect product. For $(m, n) \in M \rtimes N$, define the degeneracy and face maps as

$$
\begin{aligned}
& d_{0}: M \rtimes N \longrightarrow N \\
& (m, n) \longmapsto n \\
& d_{1}: M \rtimes N \longrightarrow N \\
& (m, n) \longmapsto\left(\partial_{1}(m)\right) n \\
& s_{0}: \quad N \quad \longrightarrow M \rtimes N \\
& n \quad \longmapsto(1, n) \text {. }
\end{aligned}
$$

Now by the actions of $M$ and $N$ on $L$ we obtain the $H_{2}=(L \rtimes M) \rtimes(M \rtimes N)$ semidirect product. For $l \in L, m, m^{\prime} \in M, n \in N$, define the degeneracy and face maps as

$$
\begin{aligned}
& d_{0}:(L \rtimes M) \rtimes(M \rtimes N) \longrightarrow(M \rtimes N) \\
& \left(l, m, m^{\prime}, n\right) \quad \longmapsto\left(m^{\prime}, n\right), \\
& d_{1}:(L \rtimes M) \rtimes(M \rtimes N) \longrightarrow(M \rtimes N) \\
& \left(l, m, m^{\prime}, n\right) \quad \longmapsto\left(m m^{\prime}, n\right), \\
& d_{2}:(L \rtimes M) \rtimes(M \rtimes N) \quad \longrightarrow(M \rtimes N) \\
& \left(l, m, m^{\prime}, n\right) \quad \longmapsto\left(\partial_{2}(l) m, \partial_{1}\left(m^{\prime}\right) n\right), \\
& s_{0}:(M \rtimes N) \quad \longrightarrow(L \rtimes M) \rtimes(M \rtimes N) \\
& \left(m^{\prime}, n\right) \quad \longmapsto\left(1,1, m^{\prime}, n\right), \\
& s_{1}:(M \rtimes N) \quad \longrightarrow(L \rtimes M) \rtimes(M \rtimes N) \text {, } \\
& \left(m^{\prime}, n\right) \quad \longmapsto\left(1, m^{\prime}, 1, n\right) .
\end{aligned}
$$

Since $\{,\}_{(2)(1)}$ is a 2 -crossed module there is an action of $L$ on $K$ defined as

$$
{ }^{l} k=\left\{\partial_{3} k, l\right\}_{(2)(1)} k^{-1}
$$

for $l \in L, k \in K$. Using this action we obtain a semidirect product $K \rtimes L$. The action of $(l, m) \in L \rtimes M$ on $(k, l) \in K \rtimes L$ can be expressed as

$$
\begin{aligned}
{ }^{(1, m)}\left(k, l^{\prime}\right) & =\left({ }^{m}\left({ }^{1} k\right),{ }^{m}\left({ }^{1} l^{\prime}\right)\right) \\
& =\left({ }^{m}(k),{ }^{m}\left(l^{\prime}\right)\right), \\
{ }^{(l, 1)}\left(k, l^{\prime}\right) & =\left({ }^{1}\left({ }^{l} k\right),{ }^{1}\left({ }^{l} l^{\prime}\right)\right) \\
& =\left({ }^{l} k,{ }^{l} l^{\prime}\right) \\
& =\left({ }^{\partial_{2} l} k\left\{l, \partial_{3} k\right\}_{(2)(1)}, l l^{\prime} l^{-1}\right) .
\end{aligned}
$$

After these definitions we have the semidirect product

$$
H_{3}=(K \rtimes L) \rtimes(L \rtimes M) \rtimes(M \rtimes N) .
$$


Define the degeneracy and face maps as:

$$
\begin{aligned}
& d_{0}: \quad(K \rtimes L) \rtimes(L \rtimes M) \rtimes(M \rtimes N) \quad \longrightarrow \quad(L \rtimes M) \rtimes(M \rtimes N) \\
& \left(k, l, l^{\prime}, m, m^{\prime}, n\right) \quad \longmapsto \quad\left(l^{\prime}, m, m^{\prime}, n\right) \text {, } \\
& d_{1}: \quad(K \rtimes L) \rtimes(L \rtimes M) \rtimes(M \rtimes N) \longrightarrow(L \rtimes M) \rtimes(M \rtimes N) \\
& \left(k, l, l^{\prime}, m, m^{\prime}, n\right) \quad \longmapsto \quad\left(l, m, m^{\prime}, n\right), \\
& d_{2}: \quad(K \rtimes L) \rtimes(L \rtimes M) \rtimes(M \rtimes N) \longrightarrow(L \rtimes M) \rtimes(M \rtimes N) \\
& \left(k, l, l^{\prime}, m, m^{\prime}, n\right) \quad \longmapsto \quad\left(l l^{\prime}, m, m^{\prime}, n\right), \\
& d_{3}: \quad(K \rtimes L) \rtimes(L \rtimes M) \rtimes(M \rtimes N) \longrightarrow(L \rtimes M) \rtimes(M \rtimes N) \\
& \left(k, l, l^{\prime}, m, m^{\prime}, n\right) \quad \longmapsto\left(\partial_{3} k l, \partial_{2} l^{\prime} m, m^{\prime}, n\right), \\
& s_{0}: \quad(L \rtimes M) \rtimes(M \rtimes N) \quad \longrightarrow \quad(K \rtimes L) \rtimes(L \rtimes M) \rtimes(M \rtimes N) \\
& \left(l, m, m^{\prime}, n\right) \quad \longmapsto \quad\left(1, l, 1, m, m^{\prime}, n\right), \\
& s_{1}: \quad(L \rtimes M) \rtimes(M \rtimes N) \quad \longrightarrow \quad(K \rtimes L) \rtimes(L \rtimes M) \rtimes(M \rtimes N) \\
& \left(l, m, m^{\prime}, n\right) \quad \longmapsto\left(1,1, l, m, m^{\prime}, n\right), \\
& s_{2}: \quad(L \rtimes M) \rtimes(M \rtimes N) \quad \longrightarrow(K \rtimes L) \rtimes(L \rtimes M) \rtimes(M \rtimes N) \\
& \left(l, m, m^{\prime}, n\right) \quad \longmapsto\left(1, l, 1, m, m^{\prime}, n\right) .
\end{aligned}
$$

Thus we have a 3-truncated simplicial group $\mathbf{H}=\left\{H_{0}, H_{1}, H_{2}, H_{3}\right\}$. Applying the 3skeleton functor defined in Subsection 2.1 to 3-truncation gives us a simplicial group, which will again be denoted $\mathbf{H}$, and the result has Moore complex

$$
\text { ker } \partial_{3} \rightarrow K \rightarrow L \rightarrow M \rightarrow N .
$$

We set $\mathbf{H}^{\prime}=\mathbf{s t}_{3} \mathbf{H}$ and note that $N H_{p}^{\prime}=D_{p} \cap N H_{p}$, where $D_{p}$ is the subgroup of $H_{p}$ generated by the degenerate elements, and so $N H_{p}^{\prime}=1$ if $p>4$. We claim $N H_{4}^{\prime}=1$. By Theorem B, case $n=4$ (see $[\mathbf{2 2}]$ ), $\partial_{4}\left(N H_{4} \cap D_{4}\right)$ is the product of commutators. A direct calculation, using the descriptions of the actions and the face maps above, shows that these are all trivial, so $\partial_{4}\left(N H_{4} \cap D_{4}\right)=1$, but $\partial_{4}^{\mathbf{H}}$ is a monomorphism so $\mathrm{NH}_{4}^{\prime}$ is trivial as required.

Proposition 5.3. Let $\mathbf{G}$ be a simplicial group, let $\pi_{n}^{\prime}$ be the homotopy groups of its 3 -crossed module and let $\pi_{n}$ be the homotopy groups of the classifying space of $\mathbf{G}$; then we have $\pi_{n} \cong \pi_{n}^{\prime}$ for $n=0,1,2,3,4$.

Proof. Let $\mathbf{G}$ be a simplicial group. The $n$th homotopy group of $\mathbf{G}$ is the $n$th homology of the Moore complex of $\mathbf{G}$; i.e.,

$$
\pi_{n}(\mathbf{G}) \cong H_{n}(\mathbf{N G}) \cong \frac{\operatorname{ker} d_{n-1}^{n-1} \cap N G_{n-1}}{d_{n}^{n}\left(N G_{n}\right)}
$$

Thus the homotopy groups $\pi_{n}(\mathbf{G})=\pi_{n}$ of $\mathbf{G}$ are

$$
\pi_{n}= \begin{cases}N G_{0} / d_{1}\left(N G_{1}\right) & n=1 \\ \frac{\operatorname{ker} d_{n-1}^{n-1} \cap N G_{n-1}}{d_{n}^{n}\left(N G_{n}\right)} & n=2,3,4 \\ 0 & n=0 \text { for } n>4\end{cases}
$$


and the homotopy groups $\pi_{n}^{\prime}$ of its 3 -crossed module are

$$
\pi_{n}^{\prime}= \begin{cases}N G_{0} / \partial_{1}(M) & n=1 \\ \operatorname{ker} \partial_{1} / \operatorname{Im}\left(\partial_{2}\right) & n=2 \\ \operatorname{ker} \partial_{2} / \operatorname{Im}\left(\partial_{3}\right) & n=3 \\ \operatorname{ker} \partial_{3} & n=4 \\ 0 & n=0 \text { for } n>4\end{cases}
$$

The isomorphism $\pi_{n} \cong \pi_{n}^{\prime}$ can be shown by a direct calculation.

\subsection{Crossed 3-cubes}

Crossed squares (or crossed 2-cubes) were introduced by D. Guin-Waléry and J.-L. Loday [19]; see also [8] and [20].

Definition 5.4. A crossed square is a commutative diagram of group morphisms

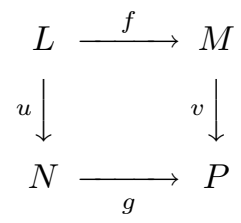

with the action of $P$ on every other group and a function $h: M \times N \rightarrow L$ such that:

(1) the maps $f$ and $u$ are $P$-equivariant and $g, v, v \circ f$ and $g \circ u$ are crossed modules,

(2) $f \circ h(x, y)=x^{g(y)} x^{-1}, u \circ h(x, y)={ }^{v(x)} y y^{-1}$,

(3) $h(f(z), y)=z^{g(y)} z^{-1}, h(x, u(z))={ }^{v(x)} z z^{-1}$,

(4) $h\left(x x^{\prime}, y\right)={ }^{v(x)} h\left(x^{\prime}, y\right) h(x, y), h\left(x, y y^{\prime}\right)=h(x, y)^{g(y)} h\left(x, y^{\prime}\right)$,

(5) $h\left({ }^{t} x,{ }^{t} y\right)={ }^{t} h(x, y)$

for $x, x^{\prime} \in M, y, y^{\prime} \in N, z \in L$ and $t \in P$.

It is a consequence of the definition that $f: L \rightarrow M$ and $u: L \rightarrow N$ are crossed modules where $M$ and $N$ act on $L$ via their images in $P$. A crossed square can be seen as a crossed module in the category of crossed modules.

A crossed square can be seen as a complex of crossed modules of length one; thus Conduché $[\mathbf{1 3}]$ gave a direct proof from crossed squares to 2-crossed modules. This construction is the following:

Let

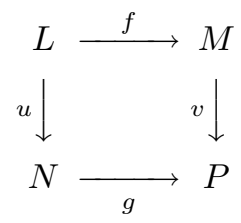

be a crossed square. Then seeing the horizontal morphisms as a complex of crossed modules, the mapping cone of this square is a 2-crossed module $L \stackrel{\partial_{2}}{\longrightarrow} M \rtimes N \stackrel{\partial_{1}}{\longrightarrow} P$, where $\partial_{2}(z)=\left(f(z)^{-1}, u(z)\right)$ for $z \in L, \partial_{1}(x, y)=g(x) g(y)$ for $x \in M$ and $y \in N$, and the Peiffer lifting is given by $\left\{(x, y),\left(x^{\prime}, y^{\prime}\right)\right\}=h\left(x, y y^{\prime} y^{-1}\right)$. 
Crossed squares were generalised by G. Ellis in $[\mathbf{1 6}, \mathbf{1 7}]$ and were called "Crossed $n$-cubes which were related to simplicial groups by T. Porter in [23]. Here we only consider this construction for $n=3$ and look at the relation between crossed 3-cubes (see Appendix B) and 3-crossed modules.

Let

$$
K \stackrel{\partial_{3}}{\longrightarrow} L \stackrel{\partial_{2}}{\longrightarrow} M \stackrel{\partial_{1}}{\longrightarrow} N
$$

be a 3-crossed module and let $\mathbf{G}$ be the corresponding simplicial group. The crossed 3-cube associated to $\mathbf{G}$, defined by T. Porter in [23], is up to a canonical isomorphism

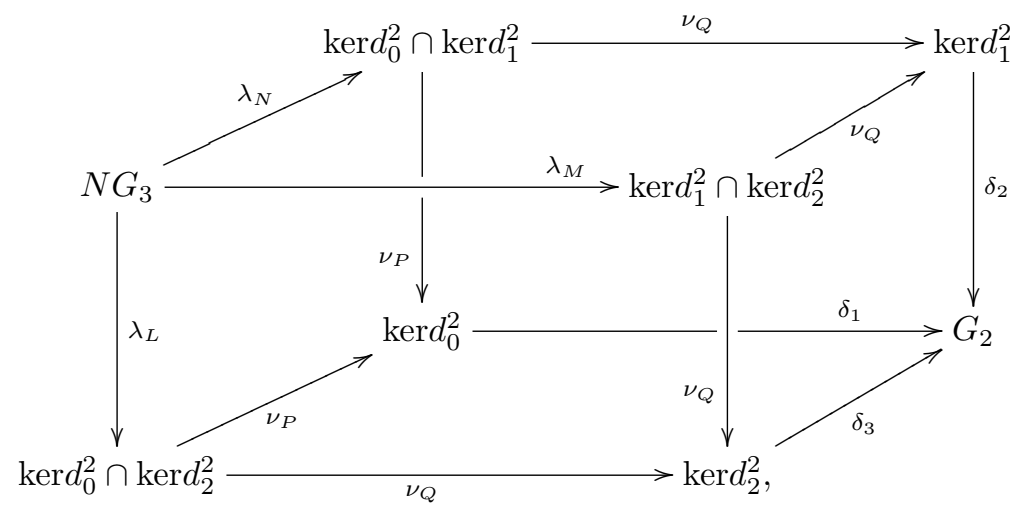

where $\lambda_{L}, \lambda_{M}, \lambda_{N}$ are restrictions of $d_{3}^{3}$ and the others are inclusions. The $h$-maps are

$$
\begin{aligned}
& h_{1}: \operatorname{ker} d_{1}^{2} \times \operatorname{ker} d_{0}^{2} \cap \operatorname{ker} d_{2}^{2} \quad \longrightarrow \quad N G_{3} \\
& (x, y) \quad \longmapsto\left[s_{1} x_{2} s_{0} x_{2}^{-1}, s_{2} y_{2}^{-1} s_{1} y_{2}^{-1}\right], \\
& h_{2}: \operatorname{ker} d_{0}^{2} \times \operatorname{ker} d_{1}^{2} \cap \operatorname{ker} d_{2}^{2} \quad \longrightarrow \quad N G_{3} \\
& (x, y) \quad \longmapsto\left[s_{1} x_{2}, s_{2} y_{2} s_{1} y_{2}^{-1} s_{0} y_{2}\right], \\
& h_{3} \text { : } \operatorname{ker} d_{0}^{2} \cap \operatorname{ker} d_{1}^{2} \times \operatorname{ker} d_{2}^{2} \quad \longrightarrow N G_{3} \\
& (x, y) \quad \longmapsto\left[s_{2} x_{2}, s_{2} y_{2} s_{1} y_{2}^{-1}\right], \\
& h_{7} \text { : } \operatorname{ker} d_{0}^{2} \cap \operatorname{ker} d_{2}^{2} \times \operatorname{ker} d_{1}^{2} \cap \operatorname{ker} d_{2}^{2} \longrightarrow N G_{3} \\
& (x, y) \quad \longmapsto h_{2}(i x, y)=h_{2}(x, y), \\
& h_{8} \text { : } \operatorname{ker} d_{0}^{2} \cap \operatorname{ker} d_{1}^{2} \times \operatorname{ker} d_{0}^{2} \cap \operatorname{ker} d_{2}^{2} \longrightarrow N G_{3} \\
& (x, y) \quad \longmapsto h_{3}(x, i y)=h_{3}(x, y), \\
& h_{9}: \operatorname{ker} d_{0}^{2} \cap \operatorname{ker} d_{1}^{2} \times \operatorname{ker} d_{1}^{2} \cap \operatorname{ker} d_{2}^{2} \quad \longrightarrow \quad N G_{3} \\
& (x, y) \quad \longmapsto h_{3}(x, i y)=h_{3}(x, y),
\end{aligned}
$$

and the others are commutators. (The name of the maps are given with respect to the crossed 3 -cube definition in $[\mathbf{1 6}]$.) 
Then in terms of the 3-crossed module, this crossed cube can be written as

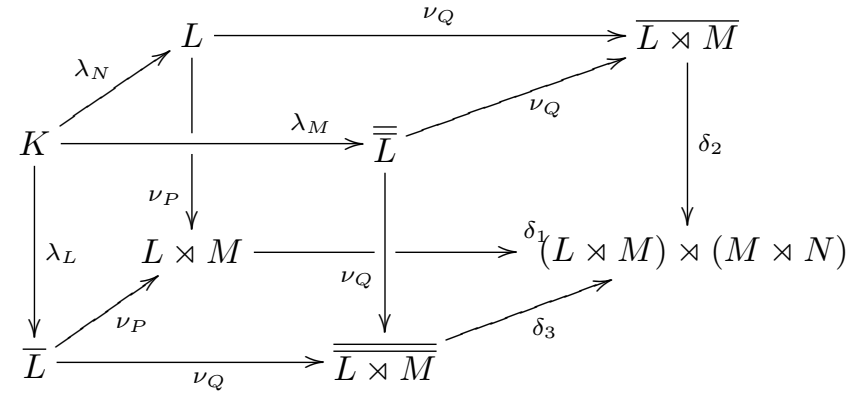

where

$$
\begin{aligned}
L \ltimes M & \cong\{(l, m, 1,1): l \in L, m \in M\}, \\
\overline{L \ltimes M} & =\left\{\left(l, m, m^{\prime}, 1\right): l \in L, m, m^{\prime} \in M, m m^{\prime}=1, l \in L, m \in M\right\}, \\
\overline{\overline{L \ltimes M}} & =\left\{\left(l, m, m^{\prime}, n\right): \partial_{2}(l)=1, \partial_{1}\left(m^{\prime}\right) n=1, l \in L, m \in M\right\}, \\
L & \cong\{(l, 1,1,1): l \in L\}, \\
\bar{L} & =\left\{(l, m, 1,1): \partial_{2} l m=1, l \in L, m \in M\right\} \\
& =\left\{\left(l, \partial_{2}\left(l^{-1}\right), 1,1\right): l \in L\right\}, \\
\overline{\bar{L}} & =\left\{\left(l, m, m^{\prime}, 1\right): m m^{\prime}=1, \partial_{2}(l) m=1, \partial_{1}\left(m^{\prime}\right) n=1, l \in L, m \in M, n \in N\right\} \\
& =\left\{\left(l, \partial_{2}\left(l^{-1}\right), \partial_{2}(l), 1\right): l \in L\right\} .
\end{aligned}
$$

By Definition 2.7, given in [13], we have the mapping cone of this crossed 3-cube as

$$
K \rightarrow(L \ltimes \overline{\bar{L}}) \ltimes \bar{L} \rightarrow(\overline{L \ltimes M})((L \ltimes M) \ltimes(\overline{\overline{L \ltimes M}})) \rightarrow(L \ltimes M) \ltimes(M \ltimes N) .
$$

Example 5.5. Let

$$
K \stackrel{\partial_{3}}{\longrightarrow} L \stackrel{\partial_{2}}{\longrightarrow} M \stackrel{\partial_{1}}{\longrightarrow} N
$$

be a 3 -crossed module. If $M=\{1\}$, then, for $i=1,2,3$, the commutative diagram

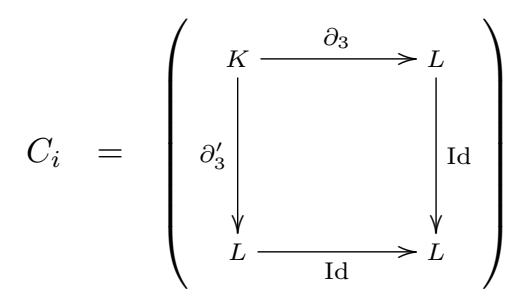

is a crossed square with the following $h_{i}$-maps:

$$
\begin{aligned}
h_{1}= & \{,\}_{(2)(1)}: L \times L \rightarrow K, \\
h_{2}= & \{,\}_{(0)(2)}: L \times L \rightarrow K, \\
h_{3}= & \{,\}_{(0)(1)}: L \times L \rightarrow K, \\
& (x, y) \mapsto\{y, x\}_{(1)(0)}^{-1},
\end{aligned}
$$

where the action of $L$ on itself is by conjugation. 
Since $M=\{1\}, \partial_{2}(l)=1_{M}$ for all $l \in L$. Thus from the 3 -crossed module axioms we find

$$
\begin{aligned}
\left\{l, \partial_{3} k\right\}_{(2)(1)} & =(l \cdot k) k^{-1}, \\
\left\{l l^{\prime}, l^{\prime \prime}\right\}_{(2)(1)} & =l \cdot\left\{l^{\prime}, l^{\prime \prime}\right\}_{(2)(1)}\left\{l, l^{\prime \prime}\right\}_{(2)(1)}, \\
\left\{l, l^{\prime} l^{\prime \prime}\right\}_{(2)(1)} & =\left\{l, l^{\prime}\right\}_{(2)(1)} l^{\prime}\left\{l, l^{\prime \prime}\right\}_{(2)(1)}, \\
\partial_{3}\left\{l, l^{\prime}\right\}_{(2)(1)} & =l\left(l^{\prime} l^{-1}\right), \\
\left(\left\{l^{\prime}, l\right\}_{(1)(0)}\right)^{-1} & =\left\{l, l^{\prime}\right\}_{(2)(1)}, \\
\left\{l, l^{\prime}\right\}_{(0)(2)} & =1
\end{aligned}
$$

for all $l, l^{\prime}, l^{\prime \prime} \in L, k, k^{\prime} \in K$. Using these equalities and the 3-crossed module axioms, crossed square conditions can be easily verified.

In this example the result is trivial for the $h$-map $h_{1}$ from $[\mathbf{1 2}]$ since $\{,\}_{(2)(1)}$ is a 2-crossed module. Here the liftings $\{,\}_{(0)(2)},\{,\}_{(0)(1)}$ are not 2-crossed modules but the associated $h$-maps are crossed squares.

Example 5.6. In the universal cube definition given in [16], take $P, R, N, M=L$, $S=M$ and $T_{0}=K \otimes K \otimes K$. Then

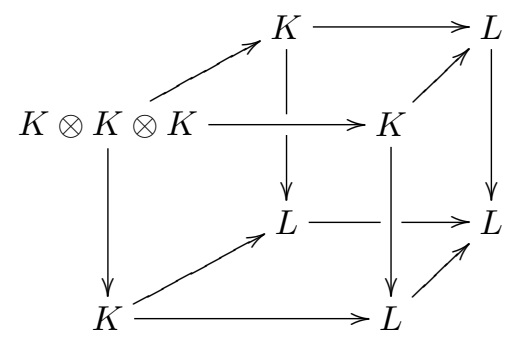

is a universal crossed 3-cube with the crossed squares obtained by the Peiffer maps $\{,\}_{(0)(1)},\{,\}_{(2)(1)},\{,\}_{(0)(2)}$ given in the above proposition.

\section{Appendix A.}

Proof of Proposition 5.1.

3CM1)

Since

$$
\begin{aligned}
\bar{\partial}_{3}\left(\left\{x_{2}, y_{2}\right\}_{(2)(1)}\right) & =\left[x_{2}, y_{2}\right]\left[y_{2}, s_{1} \partial_{2} x_{2}\right] \\
& =x_{2} y_{2} x_{2}^{-1} y_{2}^{-1} y_{2} s_{1} \partial_{2} x_{2} y_{2}^{-1} s_{1} \partial_{2} x_{2}^{-1} \\
& =x_{2} y_{2} x_{2}^{-1}\left({ }^{\partial_{2} x_{2}} y_{2}\right)^{-1}
\end{aligned}
$$

$$
d_{4}\left(F_{(1)(3,2)}\left(x_{3}, y_{2}\right)\right)=\left[s_{1} d_{3} x_{3}, s_{2} y_{2}\right]\left[s_{2} y_{2}, s_{2} d_{3} x_{3}\right]\left[x_{3}, s_{2} y_{2}\right]
$$

we find

$$
\begin{aligned}
\left\{\bar{\partial}_{3} x_{3}, y_{2}\right\}_{(2)(1)} & =\left[s_{1} \bar{\partial}_{3} x_{3}, s_{2} y_{2}\right]\left[s_{2} y_{2}, s_{2} \bar{\partial}_{3} x_{3}\right] \\
& \equiv\left[x_{3}, s_{2} y_{2}\right] \quad \bmod \partial_{4}\left(N G_{4} \cap D_{4}\right) \\
& =x_{3}\left({ }^{y_{2}} x_{3}\right)^{-1} .
\end{aligned}
$$


Since

$$
d_{4}\left(F_{(3,1)(2)}\left(x_{2}, y_{3}\right)\right)=\left[s_{1} x_{2}, s_{2} d_{3} y_{3}\right]\left[s_{2} d_{3} y_{3}, s_{2} x_{2}\right]\left[s_{2} x_{2}, y_{3}\right]\left[y_{3}, s_{1} x_{2}\right],
$$

we find

$$
\begin{aligned}
\left\{x_{2}, \bar{\partial}_{3} y_{3}\right\}_{(2)(1)}= & {\left[s_{2} x_{2}, s_{2} \bar{\partial}_{3} y_{3}\right]\left[s_{2} \bar{\partial}_{3} y_{3}, s_{1} x_{2}\right] } \\
\equiv & {\left[s_{2} x_{2}, y_{3}\right]\left[y_{3}, s_{1} x_{2}\right] \quad \bmod \partial_{4}\left(N G_{4} \cap D_{4}\right) } \\
= & { }^{x_{2}} y_{3} s_{1} x_{2} y_{3}^{-1} s_{1} x_{2}^{-1} \\
\equiv & { }^{x_{2}} y_{3}\left({ }^{\partial_{2} x_{2}} y_{3}\right)^{-1} \bmod \partial_{4}\left(N G_{4} \cap D_{4}\right), \\
\left\{x_{2} y_{2}, z_{2}\right\}_{(2)(1)}= & {\left[s_{1}\left(x_{2} y_{2}\right), s_{2} z_{2}\right]\left[s_{2} z_{2}, s_{2}\left(x_{2} y_{2}\right)\right] } \\
= & s_{1}\left(x_{2} y_{2}\right) s_{2} z_{2} s_{1}\left(x_{2} y_{2}\right)^{-1} s_{2}\left(x_{2} y_{2}\right) s_{2} z_{2}^{-1} s_{2}\left(x_{2} y_{2}\right)^{-1} \\
\equiv & s_{2}\left(x_{2} y_{2}\right) s_{2} z_{2}^{-1} s_{2}\left(x_{2} y_{2}\right)^{-1} s_{1}\left(x_{2} y_{2}\right) \\
& s_{2} z_{2} s_{1}\left(x_{2} y_{2}\right)^{-1} \bmod \partial_{4}\left(N G_{4} \cap D_{4}\right) \\
= & \left\{x_{2}, y_{2} z_{2} y_{2}^{-1}\right\}_{(2)(1)} \partial_{1} x_{2}\left\{y_{2}, z_{2}\right\}(2)(1),
\end{aligned}
$$

and

$$
\begin{aligned}
\left\{x_{2}, y_{2} z_{2}\right\}_{(2)(1)}= & {\left[s_{1}\left(x_{2}\right), s_{2}\left(y_{2} z_{2}\right)\right]\left[s_{2}\left(y_{2} z_{2}\right), s_{2}\left(x_{2}\right)\right] } \\
\equiv & {\left[s_{2}\left(x_{2}\right), s_{2}\left(y_{2} z_{2}\right)\right]\left[s_{2}\left(y_{2} z_{2}\right), s_{1}\left(x_{2}\right)\right] } \\
& \left(s_{2}\left(x_{2}\right) s_{2}\left(y_{2}\right) s_{2}\left(x_{2}\right)^{-1}\right) s_{1}\left(x_{2}\right) s_{2}\left(y_{2}\right) s_{1}\left(x_{2}\right)^{-1} \bmod \partial_{4}\left(N G_{4} \cap D_{4}\right) \\
= & \left(x_{2} y_{2} x_{2}^{-1}\right) \cdot\left\{x_{2}, z_{2}\right\}_{(2)(1)}\left\{x_{2}, y_{2}\right\}_{(2)(1)} .
\end{aligned}
$$

3CM2) Since

$$
\begin{aligned}
d_{4}\left(F_{(3,2,0)(1)}\left(x_{1}, y_{3}\right)\right)= & {\left[s_{2} s_{0} x_{1}, s_{1} d_{3} y_{3}\right]\left[s_{1} d_{3} y_{3}, s_{2} s_{1} x_{1}\right]\left[s_{2} s_{1} x_{1}, s_{2} d_{3} y_{3}\right] } \\
& {\left[s_{2} d_{3} y_{3},\right]\left[s_{2} s_{0} x_{1}, y_{3}\right]\left[y_{3}, s_{2} s_{1} x_{1}\right] } \\
d_{4}\left(F_{(3,1,0)(2)}\left(x_{1}, y_{3}\right)\right)= & {\left[s_{1} s_{0} x_{1}, s_{2} d_{3} y_{3}\right]\left[s_{2} d_{3} y_{3}, s_{2} s_{0} x_{1}\right] } \\
& {\left[s_{2} s_{0} x_{1}, y_{3}\right]\left[y_{3}, s_{1} s_{0} x_{1}\right] }
\end{aligned}
$$

and

$$
d_{4}\left(F_{(2,1,0)(3)}\left(x_{1}, y_{3}\right)\right)=\left[s_{2} s_{1} s_{0} d_{1} x_{1}, y_{3}\right]\left[y_{3}, s_{1} s_{0} x_{1}\right]
$$

we find

$$
\left\{x_{1}, \bar{\partial}_{3} y_{3}\right\}_{(1,0)(2)}=\left\{x_{1}, \bar{\partial}_{3} y_{3}\right\}_{(2,0)(1)}\left\{\bar{\partial}_{3} y_{3}, x_{1}\right\}_{(0)(2,1)} y_{3}\left({ }^{{ }_{1} x_{1}} y_{3}\right)^{-1} .
$$

3CM3) and $\mathbf{3 C M 4 )}$ are left to the reader.

3CM5) Since

$$
\begin{aligned}
d_{4}\left(F_{(3,0)(2,1)}\right)= & {\left[s_{0} x_{2}, s_{2} s_{1} \partial_{2} y_{2}\right]\left[s_{2} s_{1} \partial_{2} y_{2}, s_{1} x_{2}\right]\left[s_{2} x_{2}, s_{2} s_{1} \partial_{2} y_{2}\right] } \\
& {\left[s_{1} y_{2}, s_{2} x_{2}\right]\left[s_{1} x_{2}, s_{1} y_{2}\right]\left[s_{1} y_{2}, s_{0} x_{2}\right] }
\end{aligned}
$$


we find

$$
\begin{aligned}
\left\{x_{2}, \partial_{2} y_{2}\right\}_{(0)(2,1)} & =\left[s_{0} x_{2}, s_{2} s_{1} \partial_{2} y_{2}\right]\left[s_{2} s_{1} \partial_{2} y_{2}, s_{1} x_{2}\right]\left[s_{2} x_{2}, s_{2} s_{1} \partial_{2} y_{2}\right] \\
& \equiv\left[s_{1} y_{2}, s_{2} x_{2}\right]\left[s_{1} x_{2}, s_{1} y_{2}\right]\left[s_{1} y_{2}, s_{0} x_{2}\right] \quad \bmod \partial_{4}\left(N G_{4} \cap D_{4}\right) \\
& =\left(\left\{y_{2}, x_{2}\right\}_{(1)(2)}\right)^{-1}\left\{x_{2}, y_{2}\right\}_{(1)(0)} .
\end{aligned}
$$

3CM6) Since

$$
\begin{aligned}
d_{4}\left(F_{(2,0)(3,1)}\left(x_{2}, y_{2}\right)\right)= & {\left[s_{2} s_{0} d_{2} x_{2}, s_{1} y_{2}\right]\left[s_{1} y_{2}, s_{2} s_{1} d_{2} x_{2}\right] } \\
& {\left[s_{2} s_{1} d_{2} x_{2}, s_{2} y_{2}\right]\left[s_{2} y_{2}, s_{2} s_{0} d_{2} x_{2}\right] } \\
& {\left[s_{0} x_{2}, s_{2} y_{2}\right]\left[s_{2} y_{2}, s_{1} x_{2}\right] } \\
& {\left[s_{1} x_{2}, s_{1} y_{2}\right]\left[s_{1} y_{2}, s_{0} x_{2}\right] }
\end{aligned}
$$

we find

$$
\begin{aligned}
\left\{\partial_{2} x_{2}, y_{2}\right\}_{(2,0)(1)}= & {\left[s_{2} s_{0} \partial_{2} x_{2}, s_{1} y_{2}\right]\left[s_{1} y_{2}, s_{2} s_{1} \partial_{2} x_{2}\right] } \\
& {\left[s_{2} s_{1} \partial_{2} x_{2}, s_{2} y_{2}\right]\left[s_{2} y_{2}, s_{2} s_{0} \partial_{2} x_{2}\right] } \\
\equiv & {\left[s_{0} x_{2}, s_{2} y_{2}\right]\left[s_{2} y_{2}, s_{1} x_{2}\right] } \\
= & \left\{x_{2}, y_{2}\right\}_{(0)(2)}^{-1}\left[y_{2}, x_{2}\right]\left(\left\{x_{2}, y_{2}\right\}_{(2)(1)}\right)\left\{x_{2}, y_{2}\right\}_{(1)(0)}
\end{aligned}
$$

3CM7) Since

$$
d_{4}\left(F_{(1,0)(3,2)}\left(x_{2}, y_{2}\right)\right)=\left[s_{1} s_{0} \bar{\partial}_{2} x_{2}, s_{2} y_{2}\right]\left[s_{2} y_{2}, s_{2} s_{0} \bar{\partial}_{2} x_{2}\right]\left[s_{0} x_{2}, s_{2} y_{2}\right],
$$

we find

$$
\begin{aligned}
\left\{\partial_{2} x_{2}, y_{2}\right\}_{(1,0)(2)} & =\left[s_{1} s_{0} \bar{\partial}_{2} x_{2}, s_{2} y_{2}\right]\left[s_{2} y_{2}, s_{2} s_{0} \bar{\partial}_{2} x_{2}\right] \\
& \equiv\left[s_{0} x_{2}, s_{2} y_{2}\right] \quad \bmod \quad \partial_{4}\left(N G_{4} \cap D_{4}\right) \\
& =\left\{x_{2}, y_{2}\right\}_{(0)(2)}^{-1} .
\end{aligned}
$$

3CM8)

$$
\begin{aligned}
\bar{\partial}_{3}\left(\left\{x_{2}, y_{2}\right\}_{(1)(0)}\right) & =\left[x_{2}, y_{2}\right]\left[\bar{\partial}_{3} s_{1} x_{2}, \bar{\partial}_{3} s_{1} y_{2}\right]\left[\bar{\partial}_{3} s_{1} y_{2}, \bar{\partial}_{3} s_{0} x_{2}\right] \\
& =\left[x_{2}, y_{2}\right] s_{1}\left[\partial_{2} x_{2}, \partial_{2} y_{2}\right]\left[s_{1} \partial_{2} y_{2}, s_{0} \partial_{2} x_{2}\right] \\
& =\left[x_{2}, y_{2}\right]\left\{\partial_{2} x_{2}, \partial_{2} y_{2}\right\}
\end{aligned}
$$

3CM9)

$$
\bar{\partial}_{3}\left(\left\{x_{2}, y_{2}\right\}_{(0)(2)}\right)=\bar{\partial}_{3}\left(\left\{\partial_{2} x_{2}, y_{2}\right\}_{(1,0)(2)}\right)^{-1}
$$

3CM10)

$$
\begin{aligned}
\bar{\partial}_{3}\left\{x_{2}, y_{1}\right\}_{(0)(2,1)} & =\bar{\partial}_{3}\left(\left[s_{2} s_{1} y_{1}, s_{2} x_{2}\right]\left[s_{1} x_{2}, s_{2} s_{1} y_{1}\right]\left[s_{2} s_{1} y_{1}, s_{0} x_{2}\right]\right) \\
& =\left[s_{1} y_{1}, x_{2}\right]\left[\bar{\partial}_{3} s_{1} x_{2}, s_{1} y_{1}\right]\left[s_{1} y_{1}, \bar{\partial}_{3} s_{0} x_{2}\right] \\
& ={ }^{y_{1}} x_{2} x_{2}^{-1}\left\{\partial_{2} x_{2}, y_{1}\right\} .
\end{aligned}
$$


3CM11) Since

$$
\begin{aligned}
\bar{\partial}_{3}\left\{x_{1}, y_{2}\right\}_{(1,0)(2)} & =\left[s_{0} x_{1}, y_{2}\right]\left[y_{2}, \bar{\partial}_{3} s_{1} s_{0} x_{1}\right] \\
\bar{\partial}_{3}\left\{x_{1}, y_{2}\right\}_{(1,0)(2)}\left[\bar{\partial}_{3} s_{1} s_{0} x_{1}, y_{2}\right] & =\left[s_{0} x_{1}, y_{2}\right], \\
\bar{\partial}_{3}\left\{x_{1}, y_{2}\right\}_{(2,0)(1)} & =\left[s_{0} x_{1}, y_{2}\right]\left[y_{2}, s_{1} x_{1}\right]\left[s_{1} x_{1}, \partial_{3} s_{1} y_{2}\right]\left[\partial_{3} s_{1} y_{2}, s_{0} x_{1}\right] \\
& =\left[s_{0} x_{1}, y_{2}\right]\left[y_{2}, s_{1} x_{1}\right]\left[s_{1} x_{1}, s_{1} \partial_{2} y_{2}\right]\left[s_{1} \partial_{2} y_{2}, s_{0} x_{1}\right] \\
& =\left[s_{0} x_{1}, y_{2}\right]\left[y_{2}, s_{1} x_{1}\right]\left\{x_{1}, \partial_{2} y_{2}\right\},
\end{aligned}
$$

we find

$$
\begin{aligned}
\bar{\partial}_{3}\left\{x_{1}, y_{2}\right\}_{(2,0)(1)} & =\bar{\partial}_{3}\left\{x_{1}, y_{2}\right\}_{(1,0)(2)}\left[\bar{\partial}_{3} s_{1} s_{0} x_{1}, y_{2}\right]\left[y_{2}, s_{1} x_{1}\right]\left\{x_{1}, \partial_{2} y_{2}\right\} \\
& =\bar{\partial}_{3}\left\{x_{1}, y_{2}\right\}_{(1,0)(2)}\left[s_{1} s_{0} \partial_{1} x_{1}, y_{2}\right]\left[y_{2}, s_{1} x_{1}\right]\left\{x_{1}, \partial_{2} y_{2}\right\} \\
& =\bar{\partial}_{3}\left\{x_{1}, y_{2}\right\}_{(1,0)(2)}{ }^{\partial_{1} x_{1}} y_{2}{ }^{x_{1}} y_{2}\left\{x_{1}, \partial_{2} y_{2}\right\}
\end{aligned}
$$

3CM12) Since

$$
d_{4}\left(F_{(0)(3,1)}\left(x_{3}, y_{2}\right)\right)=\left[s_{0} d_{3} x_{3}, s_{1} y_{2}\right]\left[s_{1} y_{2}, s_{1} d_{3} x_{3}\right]\left[s_{2} d_{3} x_{3}, s_{2} y_{2}\right]\left[s_{2} y_{2}, x_{3}\right],
$$

we find

$$
\begin{aligned}
\left\{\bar{\partial}_{3} x_{3}, y_{2}\right\}_{(1)(0)} & =\left[s_{0} \bar{\partial}_{3} x_{3}, s_{1} y_{2}\right]\left[s_{1} y_{2}, s_{1} \bar{\partial}_{3} x_{3}\right]\left[s_{2} \bar{\partial}_{3} x_{3}, s_{2} y_{2}\right] \\
& \equiv\left[s_{2} y_{2}, x_{3}\right] \quad \bmod \partial_{4}\left(N G_{4} \cap D_{4}\right) \\
& \left.={ }^{y_{2}} x_{3}\right) x_{3}^{-1} .
\end{aligned}
$$

Since

$$
d_{4}\left(F_{(3,0)(1)}\left(x_{2}, y_{3}\right)\right)=\left[s_{0} x_{2}, s_{1} d_{3} y_{3}\right]\left[s_{1} d_{3} y_{3}, s_{1} x_{2}\right]\left[s_{2} x_{2}, s_{2} d_{3} y_{3}\right]\left[y_{3}, s_{2} x_{2}\right],
$$

we find

$$
\begin{aligned}
\left\{x_{2}, \bar{\partial}_{3} y_{3}\right\}_{(1)(0)} & =\left[s_{0} x_{2}, s_{1} \bar{\partial}_{3} y_{3}\right]\left[s_{1} \bar{\partial}_{3} y_{3}, s_{1} x_{2}\right]\left[s_{2} x_{2}, s_{2} \bar{\partial}_{3} y_{3}\right] \\
& \equiv\left[y_{3}, s_{2} x_{2}\right] \quad \bmod \partial_{4}\left(N G_{4} \cap D_{4}\right) \\
& =y_{3}\left({ }^{x_{2}} y_{3}\right)^{-1} .
\end{aligned}
$$

3CM13) is left to the reader.

3CM14) Since

$$
d_{4}\left(F_{(0)(3,2)}\left(x_{3}, y_{2}\right)\right)=\left[s_{0} d_{3} x_{3}, s_{2} y_{2}\right]
$$

we find

$$
\begin{aligned}
\left\{\bar{\partial}_{3} x_{3}, y_{2}\right\}_{(0)(2)} & =\left[s_{0} \bar{\partial}_{3} x_{3}, s_{2} y_{2}\right] \\
& \equiv 1 \quad \bmod \partial_{4}\left(N G_{4} \cap D_{4}\right) .
\end{aligned}
$$

3CM15) Since

$$
d_{4}\left(F_{(3,0)(2)}\left(x_{2}, y_{3}\right)\right)=\left[s_{0} x_{2}, s_{2} d_{3} y_{3}\right]\left[y_{3}, s_{0} x_{2}\right]
$$

and

$$
d_{4}\left(F_{(1,0)(2)}\left(x_{2}, y_{3}\right)\right)=\left[s_{1} s_{0} \partial_{2} x_{2}, s_{2} \partial_{3} y_{3}\right]\left[s_{2} \partial_{3} y_{3}, s_{2} s_{0} \partial_{2} x_{2}\right]\left[s_{0} x_{2}, y_{3}\right],
$$


we find

$$
\begin{aligned}
\left\{x_{2}, \bar{\partial}_{3} y_{3}\right\}_{(0)(2)} & =\left[s_{0} x_{2}, s_{2} \bar{\partial}_{3} y_{3}\right] \\
& \equiv\left[y_{3}, s_{0} x_{2}\right] \quad \bmod \partial_{4}\left(N G_{4} \cap D_{4}\right) \\
& \equiv\left[s_{2} s_{0} \partial_{2} x_{2}, s_{2} \bar{\partial}_{3} y_{3}\right]\left[s_{2} \bar{\partial}_{3} y_{3}, s_{1} s_{0} \partial_{2} x_{2}\right] \quad \bmod \partial_{4}\left(N G_{4} \cap D_{4}\right) \\
& =\left\{\partial_{2}\left(x_{2}\right), \bar{\partial}_{3}\left(y_{3}\right)\right\}_{(1,0)(2)}^{-1} .
\end{aligned}
$$

3CM16) Since

$$
\begin{aligned}
d_{4}\left(F_{(2,0)(1)}\left(x_{2}, y_{3}\right)\right)= & {\left[s_{2} s_{0} \partial_{2} x_{2}, s_{1} \partial_{3} y_{3}\right]\left[s_{1} \partial_{3} y_{3}, s_{2} s_{1} \partial_{2} x_{2}\right] } \\
& {\left[s_{2} s_{1} \partial_{2} x_{2}, s_{2} \partial_{3} y_{3}\right]\left[s_{2} \partial_{3} y_{3}, s_{2} s_{0} \partial_{2} x_{2}\right] } \\
& {\left[s_{0} x_{2}, y_{3}\right]\left[y_{3}, s_{1} x_{1}\right] }
\end{aligned}
$$

we find

$$
\begin{aligned}
\left\{\partial_{2} x_{2}, \bar{\partial}_{3} y_{3}\right\}_{(2,0)(1)}= & {\left[s_{2} s_{0} \partial_{2} x_{2}, s_{1} \bar{\partial}_{3} y_{3}\right]\left[s_{1} \bar{\partial}_{3} y_{3}, s_{2} s_{1} \partial_{2} x_{2}\right] } \\
& {\left[s_{2} s_{1} \partial_{2} x_{2}, s_{2} \bar{\partial}_{3} y_{3}\right]\left[s_{2} \bar{\partial}_{3} y_{3}, s_{2} s_{0} \partial_{2} x_{2}\right] } \\
\equiv & {\left[s_{0} x_{2}, y_{3}\right]\left[y_{3}, s_{1} x_{1}\right] \quad \bmod \partial_{4}\left(N G_{4} \cap D_{4}\right) } \\
\equiv & {\left[s_{0} x_{2}, y_{3}\right] y_{3}\left({ }_{2} x_{2} y_{3}\right)^{-1} \quad \bmod \partial_{4}\left(N G_{4} \cap D_{4}\right) } \\
\equiv & {\left[s_{1} s_{0} \partial_{2} x_{2}, s_{2} \bar{\partial}_{3} y_{3}\right]\left[s_{2} \bar{\partial}_{3} y_{3}, s_{2} s_{0} \partial_{2} x_{2}\right] \quad \bmod \partial_{4}\left(N G_{4} \cap D_{4}\right) } \\
= & \left\{\partial_{2} x_{2}, \bar{\partial}_{3} y_{3}\right\}_{(1,0)(2)} .
\end{aligned}
$$

3CM17) Since

$$
\begin{aligned}
d_{4}\left(F_{(0)(2,1)}\left(x_{3}, y_{2}\right)\right)= & {\left[s_{0} d_{3} x_{3}, s_{2} s_{1} d_{2} y_{2}\right]\left[s_{2} s_{1} d_{2} y_{2}, s_{1} d_{3} x_{3}\right] } \\
& {\left[s_{2} d_{3} x_{3}, s_{2} s_{1} d_{2} y_{2}\right]\left[s_{1} y_{2}, x_{3}\right] }
\end{aligned}
$$

and

$$
d_{4}\left(F_{(0)(2,1)}\left(x_{2}, y_{3}\right)\right)=\left[s_{2} s_{1} d_{2} x_{2}, y_{3}\right]\left[y_{3}, s_{1} x_{2}\right]
$$

we find

$$
\begin{aligned}
\left\{\bar{\partial}_{3} x_{3}, \partial_{2} y_{2}\right\}_{(0)(2,1)}= & {\left[s_{0} \bar{\partial}_{3} x_{3}, s_{2} s_{1} \partial_{2} y_{2}\right]\left[s_{2} s_{1} \partial_{2} y_{2}, s_{1} \bar{\partial}_{3} x_{3}\right] } \\
& {\left[s_{2} \bar{\partial}_{3} x_{3}, s_{2} s_{1} \partial_{2} y_{2}\right] } \\
\equiv & {\left[s_{1} y_{2}, x_{3}\right] \quad \bmod \partial_{4}\left(N G_{4} \cap D_{4}\right) } \\
\equiv & {\left[x_{3}, s_{2} s_{1} \partial_{2} y_{2}\right] \quad \bmod \partial_{4}\left(N G_{4} \cap D_{4}\right) } \\
& =x_{3}\left({ }^{\partial_{2} y_{2}} x_{3}\right)^{-1} .
\end{aligned}
$$

3CM18)

$$
\begin{aligned}
\partial_{2}\left\{x_{1}, y_{1}\right\} & =\left[x_{1}, y_{1}\right]\left[y_{1}, \partial_{2} s_{0} x_{1}\right] \\
& =x_{1} y_{1} x_{1}^{-1}\left(\partial_{1} x_{1 y_{1}}\right)^{-1} .
\end{aligned}
$$




\section{Appendix B.}

\section{B.1. Crossed 3-cube}

Given a commutative diagram of groups

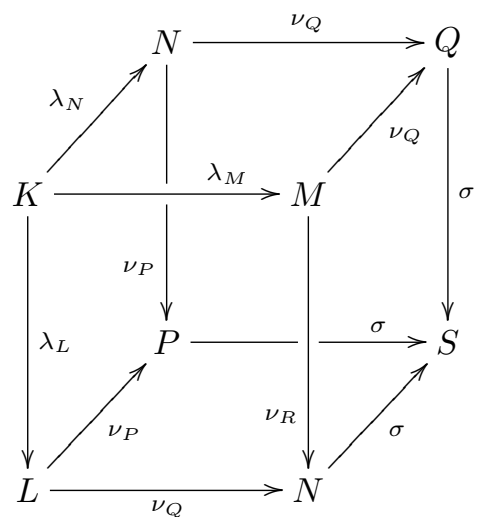

in which there is a group action of $S$ on each of the other seven groups (hence the eight groups act on each other via the action of $S$ ), and there are six functions

$$
\begin{aligned}
& h_{1}: Q \times L \longrightarrow K, \\
& h_{2}: P \times M \longrightarrow K, \\
& h_{3}: \quad N \times R \longrightarrow K, \\
& h_{4}: \quad P \times R \longrightarrow L, \\
& h_{5}: Q \times R \longrightarrow M, \\
& h_{6}: \quad P \times Q \longrightarrow N,
\end{aligned}
$$

we say that this structure is a crossed 3-cube of groups if

1. Each of the nine squares
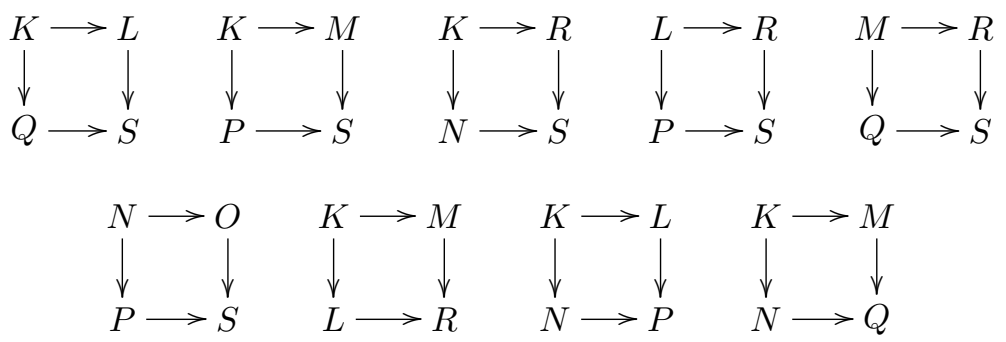

is a crossed square; for the last three squares, the functions $h: L \times M \rightarrow K$, $h: N \times L \rightarrow K, h: N \times M \rightarrow K$ are respectively given by $h(l, m)=h\left(v_{P} l, n\right)$, $h(n, l)=h\left(n, v_{R} l\right), h(n, m)=h\left(\left(n, v_{R} m\right)\right.$.

2 .

$$
\begin{gathered}
h\left(\left(v_{P} n\right)\left(v_{P} l\right), m\right) h\left(\left(v_{Q} m\right)\left(v_{Q} n\right), l\right)=h\left(n,\left(v_{R} l\right)\left(v_{R} m\right)\right) . \\
{ }^{q} h\left(h\left(p, q^{-1}\right)^{-1}, r\right)={ }^{p} h\left(q, h\left(p^{-1}, r\right)\right)^{r} h\left(p, h\left(q, r^{-1}\right)^{-1}\right) .
\end{gathered}
$$

4. 
5.

$$
\begin{aligned}
\lambda_{L} h(p, m) & =h\left(p, v_{R} m\right), \\
\lambda_{L} h(n, r) & =h\left(v_{P} n, r\right), \\
\lambda_{M} h(q, l) & =h\left(q, v_{R} l\right), \\
\lambda_{M} h(n, r) & =h\left(v_{Q} n, r\right), \\
\lambda_{N} h(p, m) & =h\left(p, v_{Q} m\right), \\
\lambda_{N} h(q, l) & =h\left(v_{P} l, q\right)^{-1} . \\
h\left(v_{Q} m, l\right) & =h\left(v_{P} l, m\right)^{-1}, \\
h\left(n, v_{R} l\right) & =h\left(v_{Q} n, l\right), \\
h\left(n, v_{R} m\right) & =h\left(v_{P} n, m\right),
\end{aligned}
$$

for all $l \in L, m \in M, n \in N, p \in P, q \in Q, r \in R$.

\section{References}

[1] Z. Arvasi and T. Porter, Higher-dimensional Peiffer elements in simplicial commutative algebras, Theory Appl. Categ. 3 (1997), no. 1, 1-23.

[2] Z. Arvasi and E. Ulualan, On algebraic models for homotopy 3-types, J. Homotopy Relat. Struct. 1 (2006), no. 1, 1-27.

[3] I. Akça and Z. Arvasi, Simplicial and crossed Lie algebras, Homology, Homotopy and Appl. 4 (2002), no. 1, 43-57.

[4] H.J. Baues, Combinatorial homotopy and 4-dimensional complexes, de Gruyter Exp. Math. 2, Walter de Gruyter \& Co., Berlin (1991).

[5] H.J. Baues, Homotopy types, in Handbook of algebraic topology (I.M. James, ed.), 1-72, North-Holland, Amsterdam (1995).

[6] D. Bourn, Moore normalization and Dold-Kan theorem for semi-abelian categories, in Categories in algebra, geometry and mathematical physics, 105-124, Contemp. Math. 431, A.M.S., Providence, RI (2007).

[7] R. Brown and N.D. Gilbert, Algebraic models of 3-types and automorphism structures for crossed modules, Proc. London Math. Soc. (3) 59 (1989), no. 3, $51-73$.

[8] R. Brown and J.-L. Loday, Van Kampen theorems for diagrams of spaces, Topology 26 (1987), no. 3, 311-335.

[9] P. Carrasco, Complejos hipercruzados, cohomologia y extensiones, Ph.D. Thesis, Universidad de Granada (1987).

[10] P. Carrasco and A.M. Cegarra, Group-theoretic algebraic models for homotopy types, J. Pure Appl. Alg. 75 (1991), no. 3, 195-235.

[11] J.L. Castiglioni and M. Ladra, Peiffer elements in simplicial groups and algebras, J. Pure Appl. Alg. 212 (2008), no. 9, 2115-2128.

[12] D. Conduché, Modules croisés généralisés de longueur 2, J. Pure Appl. Alg. 34 (1984), no. 2-3, 155-178.

[13] D. Conduché, Simplicial crossed modules and mapping cones, Georgian Math. J. 10 (2003), no. 4, 623-636. 
[14] E.B. Curtis, Simplicial homotopy theory, Adv. in Math. 6 (1971), 107-209.

[15] J. Duskin, Simplicial methods and the interpretation of "triple" cohomology, Memoirs Amer. Math. Soc. 3, no. 2, A.M.S., Providence, RI (1975).

[16] G.J. Ellis, Crossed modules and their higher dimensional analogues, Ph.D. Thesis, U.C.N.W. (1984).

[17] G.J. Ellis and R. Steiner, Higher-dimensional crossed modules and the homotopy groups of $(n+1)$-ads, J. Pure Appl. Alg. 46 (1987), no. 2-3, 117-136.

[18] P.G. Glenn, Realization of cohomology classes in arbitrary exact categories, J. Pure Appl. Alg. 25 (1982), no. 1, 33-105.

[19] D. Guin-Waléry and J.-L. Loday, Obstructions à l'excision en $K$-théorie algébrique, in Algebraic K-theory (Northwestern Univ., Evanston, Ill., 1980), 179-216, Lecture Notes in Math. 854, Springer-Verlag, New York (1981).

[20] J.-L. Loday, Spaces with finitely many nontrivial homotopy groups, J. Pure Appl. Alg. 24 (1982), 179-202.

[21] J.P. May, Simplicial objects in algebraic topology, Van Nostrand Math. Studies 11, D. Van Nostrand Co., Princeton, NJ (1967).

[22] A. Mutlu and T. Porter, Applications of Peiffer pairing in the Moore complex of a simplicial group, Theory and Appl. Categ. 4 (1998), no. 7, 148-173.

[23] T. Porter, $n$-types of simplicial groups and crossed $n$-cubes, Topology 32 (1993), no. $1,5-24$.

[24] D.M. Roberts and U. Schreiber, The inner automorphism 3-group of a strict 2-group, J. Homotopy Relat. Struct. 3 (2008), no. 1, 193-244.

[25] J.H.C. Whitehead, Combinatorial homotopy I and II, Bull. Amer. Math. Soc. 55 (1940), 213-245 and 496-543.

Z. Arvasi zarvasi@ogu.edu.tr

Department of Mathematics and Computer Sciences, Osmangazi University, Art and Science Faculty, Eskişehir, Turkey

T.S. Kuzpinari stufan@aksaray.edu.tr

Department of Mathematics, Aksaray University, Art and Science Faculty, Aksaray, Turkey

E.Ö. Uslu euslu@aku.edu.tr

Department of Mathematics, Afyon Kocatepe University, Art and Science Faculty, Afyonkarahisar, Turkey 